KCL-TH-03-02

September 8, 2018

\title{
On higher-order corrections in M theory
}

\author{
P.S. Howe and D. Tsimpis \\ Department of Mathematics \\ King's College, London
}

\begin{abstract}
A theoretical analysis of higher-order corrections to $D=11$ supergravity is given in a superspace framework. It is shown that any deformation of $D=11$ supergravity for which the lowest-dimensional component of the four-form $G_{4}$ vanishes is trivial. This implies that the equations of motion of $D=11$ supergravity are specified by an element of a certain spinorial cohomology group and generalises previous results obtained using spinorial or pure spinor cohomology to the fully non-linear theory. The first deformation of the theory is given by an element of a different spinorial cohomology group with coefficients which are local tensorial functions of the massless supergravity fields. The four-form Bianchi Identities are solved, to first order and at dimension $-\frac{1}{2}$, in the case that the lowest-dimensional component of $G_{4}$ is nonzero. Moreover, it is shown how one can calculate the first-order correction to the dimension-zero torsion and thus to the supergravity equations of motion given an explicit expression for this object in terms of the supergravity fields. The version of the theory with both a four-form and a seven-form is discussed in the presence of the five-brane anomaly-cancelling term. It is shown that the supersymmetric completion of this term exists and it is argued that it is the unique anomaly-cancelling invariant at this dimension which is at least quartic in the fields. This implies that the first deformation of the theory is completely determined by the anomaly term from which one can, in principle, read off the corrections to all of the superspace field strength tensors.
\end{abstract}




\section{Introduction}

Although $M$-theory has been around for some time now, it is still not clear precisely what the theory is, although there have been many suggestions [1]. In many situations, however, it would be sufficient to know something about the corrections to the effective theory of massless fields. In the absence of an underlying perturbative analogue of string theory such corrections cannot be found systematically, even in principle, but we might hope that supersymmetry would be sufficient to determine the first-order corrections and perhaps something beyond that. This is the subject matter of the present paper: we show that, by taking the lowest-dimensional component of the closed superspace four-form $G_{4}$ to vanish, we automatically arrive at the standard supergravity equations. This result can be rephrased in terms of a certain spinorial cohomology group and is the generalisation to the non-linear theory of the result proved at the linearised level by CNT [2] and Berkovits in [3]. We also show that the first deformation to the lowest-order theory is given by an element of another spinorial cohomology group, although with restricted coefficients. We go on to solve the four-form Bianchi identities, to first order and at dimension one-half, given an arbitrary non-zero $G_{\alpha \beta \gamma \delta}$. In particular, we show how one can construct the first-order correction to the dimension-zero component of the torsion tensor in this fashion. We also show that this torsion automatically satisfies the constraints imposed by the first geometrical Bianchi identity. We then comment on the four-form/seven-form formulation of the theory in the presence of anomalies. We construct (in principle) an invariant which includes the fivebrane anomaly-cancelling Chern-Simons term and argue that this is the only invariant, apart from the classical action, which has the same or lower dimension than $R^{4}$ and which is at least quartic in the fields. It is also argued that, at this order, all the components of the superspace field strength tensors can be determined systematically using the Bianchi identities and the input of the anomaly term.

There have been many papers on higher-order corrections in supergravity and string theory. Some relevant papers are $[4,5,6,7,9,10,11,12]$ and [13] the last of which contains a review and further references. However, the construction of the complete $R^{4}$ terms in type II string theory or in M-theory is (and remains) a difficult problem. A recent attempt was made in [14] to construct an $R^{4}$ action in type IIB based on a chiral measure in on-shell IIB superspace, but it was subsequently observed that such a measure does not exist [15]. A completely different recent approach was taken by the authors of [13] who used partial results from type II string theory and attempted to lift them to eleven dimensions, but this proved to be too difficult to carry out completely.

There have also been several papers on the subject directly in $D=11$ superspace. We recall that the equations of motion of $D=11$ supergravity, discovered in [16], were recast in superspace form in references $[17,18]$. In the first of these, the superspace constraints were constructed from the component theory by the method of gauge completion, while in the second, they were found from the supergravity multiplet by dimensional analysis. At the time no attempt was made to extract a minimal set of constraints, but there have subsequently been a number of papers on this subject. In [19] one of the present authors showed that the equations of motion could be understood as integrability conditions for a certain differential operator in membrane superspace (the space of maps from a two-dimensional spatial membrane to the $D=11$ superspace). This 
approach makes use of pure spinors, i.e. complex bosonic spinors $u^{\alpha}$ satisfying

$$
u^{\alpha}\left(\gamma^{a}\right)_{\alpha \beta} u^{\beta}=u^{\alpha}\left(\gamma^{a b}\right)_{\alpha \beta} u^{\beta}=0
$$

The integrability conditions lead to constraints on both $T_{\alpha \beta}{ }^{c}$ and $G_{\alpha \beta C D}$ which together imply the equations of motion, although again this is a redundant set. Explicitly, the constraints found this way are

$$
\left(\gamma_{a b c d e}\right)^{\alpha \beta} T_{\alpha \beta}^{f}=\left(\gamma_{a b c d e}\right)^{\alpha \beta} G_{\alpha \beta C D}=0
$$

Somewhat later, it was shown that it is only necessary to consider the geometrical part of the theory and to set

$$
T_{\alpha \beta}^{c}=-i\left(\gamma^{c}\right)_{\alpha \beta}
$$

to obtain the supergravity equations of motion [20]. This result is most easily established in Weyl superspace in which the structure group is taken to include a scale factor.

On the basis of this information an extensive analysis of the geometrical Bianchi identities was carried out in [21] for the case of a partially-modified $T_{\alpha \beta}{ }^{c}$. A complete investigation is in progress [22]. A discussion of the relevant spinorial cohomology groups with unrestricted coefficients was given in [2]. In [21] it was observed that, if the negative dimension components of $G_{A B C D}$ are taken to vanish, then the resulting theory cannot generate M-theory corrections. This point was discussed in more detail in [23].

In a recent paper, in which he attempted to generalise his pure spinor approach to string theory to the supermembrane, Berkovits [3] discussed the quantisation of the $D=11$ superparticle using a BRST operator of the form $Q:=u^{\alpha} D_{\alpha}$ where $u^{\alpha}$ is taken to satisfy only the first constraint of (1), i.e. $u \gamma^{a} u=0$, and where $D_{\alpha}$ is the flat superspace derivative. ${ }^{1}$ He showed that the linearised equations of motion of $D=11$ supergravity are equivalent to finding an element of the ghost number three cohomology group of $Q$ where $u$ is assigned ghost number one. One of the results obtained in the present paper (section four) is a proof that any deformation of the $D=11$ supergravity equations of motion for which $G_{\alpha \beta \gamma \delta}=0$ is trivial which is essentially a non-linear reformulation of the $\mathrm{CNT} /$ Berkovits result. To see this one needs to know a little bit about spinorial cohomology and this is discussed in a geometrical framework in the next section. The notion of spinorial cohomology was introduced in $[26,2]$ and was used in explicit investigations of ten-dimensional SYM in $[25,24]$. In these references it was pointed out that supersymmetric deformations can be understood perturbatively as elements in the cohomology of an appropriately defined nilpotent spinor operator. The latter was expressed in terms of a supersymmetic spinor derivative followed by a projection onto the highest-weight representation. The relation between spinorial and pure spinor cohomologies is discussed in Appendix D.

In section four we consider the $d G_{4}=0$ Bianchi identities in the case that $G_{\alpha \beta \gamma \delta} \neq 0$ and show how one may obtain the dimension zero component of the torsion tensor in terms of derivatives

\footnotetext{
${ }^{1}$ We shall refer to such an object as a B-pure spinor to distinguish it from a pure spinor in Cartan's sense which obeys (1) in $D=11$.
} 
of this object by this means. Further details of the calculation are given in section six. In section five we discuss what happens when the seven-form field strength is included. We show how to construct an action which is fully supersymmetric and which includes the Chern-Simons term necessary to cancel the fivebrane anomaly. Appendices A, B and C cover some technical details of the four-form Bianchi identity calculations.

\section{Supergravity}

We recall that $D=11$ supergravity can be described à la Wess-Zumino in a superspace with eleven even and thirty-two odd dimenions in which the structure group is taken to be the elevendimensional spin group. The preferred coframes are denoted $E^{A}=\left(E^{a}, E^{\alpha}\right)$ where $a=0, \ldots 10$ is a Lorentz vector index and $\alpha=1, \ldots 32$ is a spinor index, the spinors being Majorana. The dual frame basis of vector fields is denoted by $E_{A}=\left(E_{a}, E_{\alpha}\right)$. The preferred coframes are related to the coordinate basis by the supervielbein, $E^{A}=d z^{M} E_{M}{ }^{A}$. Note that the splitting of the tangent space into even and odd is respected by the structure group. We also introduce a connection one-form $\Omega_{A}{ }^{B}$ which takes its values in the Lie algebra of the Lorentz group and define the torsion and curvature in the usual way:

$$
\begin{aligned}
T^{A} & =D E^{A}:=d E^{A}+E^{B} \Omega_{B}{ }^{A}=\frac{1}{2} E^{C} E^{B} T_{B C}{ }^{A} \\
R_{A}{ }^{B} & =d \Omega_{A}{ }^{B}+\Omega_{A}{ }^{C} \Omega_{C}{ }^{B}=\frac{1}{2} E^{D} E^{C} R_{C D, A}{ }^{B}
\end{aligned}
$$

From the definitions we have the Bianchi identities $D T^{A}=E^{B} R_{B}{ }^{A}$ and $D T^{A}=0$. The assumption that the structure group is the Lorentz group implies that $R_{a}{ }^{\beta}=R_{\alpha}{ }^{b}=0$ while

$$
R_{\alpha}^{\beta}=\frac{1}{4}\left(\gamma^{a b}\right)_{\alpha}^{\beta} R_{a b}
$$

We also require that we have supersymmetry, so that the dimension zero torsion tensor should have the form

$$
T_{\alpha \beta}{ }^{c}=-i\left(\left(\gamma^{c}\right)_{\alpha \beta}+\left(\gamma^{d_{1} d_{2}}\right)_{\alpha \beta} X_{d_{1} d_{2}}{ }^{c}+\left(\gamma^{d_{1} d_{2} d_{3} d_{4} d_{5}}\right)_{\alpha \beta} Y_{d_{1} d_{2} d_{3} d_{4} d_{5}}{ }^{c}\right)
$$

where the tensors $X$ and $Y$ are traceless and have vanishing totally antisymmetric parts. Note that these constraints on $T_{\alpha \beta}^{c}$ can be imposed by suitable field redefinitions of the supervielbein $[27]$.

Given this basic structure Dragon's theorem [28] implies that the components of the curvature tensor can be computed from the components of the torsion tensor from the first Bianchi identity while the second Bianchi identity is automatically satisfied if the first one is. In index notation the first identity reads

$$
I_{A B C}^{D}:=\nabla_{[A} T_{B C]}^{D}+T_{[A B}^{E} T_{|E| C]}^{D}-R_{[A B, C]}^{D}
$$


where the square brackets denote graded antisymmetrisation.

If we take the dimension-zero tensors $X$ and $Y$ to be zero, then the Bianchi identities imply that there is a single spinor superfield at dimension one-half after all the permissible field redefinitions have been carried out [29]. However, this superfield is in fact a derivative of a scalar superfield which can be removed by a rescaling of the supervielbein. This can be most easily seen by including scale transformations in the structure group and modifying the connection and curvature [20]. One can then show that taking the dimension zero torsion to be $-i\left(\gamma^{c}\right)_{\alpha \beta}$ implies that there are no dimension one-half fields (because we have the freedom to choose an extra conventional constraint corresponding to the scale part of the connection), and furthermore that the scale part of the curvature vanishes. We can therefore set the scale connection to zero and return to a Lorentzian superspace which will now have no dimension one-half field present. The fields that one is left with are those of the on-shell supergravity theory which must (and do) obey their equations of motion by virtue of supersymmetry.

We recall that these fields are the graviton, the gravitino and a three-form potential. Since these are all gauge fields they are visible in the components of the torsion tensor through their field strengths. The field strengths of the gravitino and curvature can be identified with the leading components of the superspace torsion tensor at dimension three-halves, $T_{a b}{ }^{\gamma}$, and the curvature tensor at dimension two, $R_{a b, c}{ }^{d}$, respectively. The dimension-one field strength of the three-form potential, which we shall denote by $W_{a b c d}$, appears in the dimension-one components of both the torsion tensor and the curvature:

$$
T_{a \beta}^{\gamma}=-\frac{1}{36}\left(\left(\gamma^{b c d}\right)_{\beta}{ }^{\gamma} W_{a b c d}+\frac{1}{8}\left(\gamma_{a b c d e}\right)_{\beta}{ }^{\gamma} W^{a b c d}\right)
$$

and

$$
R_{\alpha \beta, a b}=\frac{i}{6}\left(\left(\gamma^{c d}\right)_{\alpha \beta} W_{a b c d}+\frac{1}{24}\left(\gamma_{a b c d e f}\right)_{\alpha \beta} W^{c d e f}\right)
$$

Furthermore, the independent components of the superfield $W$ are precisely the field strengths of the component fields of the supergravity multiplet.

At this point, we have not located the three-form potential, but we can construct a closed superspace four-form $G_{4}$ whose only non-vanishing components are $G_{a b c d}=W_{a b c d}$ and

$$
G_{\alpha \beta c d}=-i\left(\gamma_{c d}\right)_{\alpha \beta}
$$

The fact that this $G_{4}$ satisfies $d G_{4}=0$ does not require any new information that we have not met before in the analysis of the Bianchi identities for the torsion and curvature. We therefore deduce the existence of a three-form potential $C$ such that $G_{4}=d C$.

To summarise, then, we have seen that the equations of motion of $D=11$ supergravity, as well as the component field content of the theory, follow from the constraint $T_{\alpha \beta}{ }^{c}=-i\left(\gamma^{c}\right)_{\alpha \beta}$. Furthermore, if we were to take a minimalist point of view, we could in principle dispense with the connection formalism and just consider $T_{\alpha \beta}{ }^{c}$ on its own. However, we shall not pursue this 
approach since it is more convenient to use the usual connection formalism especially when one wishes to consider higher-order corrections.

\section{Spinorial cohomology}

In this section we shall give a geometrical definition of spinorial cohomology. In order to define such a concept one has to choose an odd tangent bundle $F$. We shall also suppose that we have the usual machinery of supergravity, i.e. Lorentzian structure group, connection, torsion and curvature as discussed above although it is possible to avoid much of this. In particular we suppose that the tangent bundle $T$ is a direct sum of the odd and even bundles. In making a choice of the complementary even sub-bundle $B$ we are free to fix a conventional constraint on the dimension one-half torsion. A natural choice would be

$$
\left(\gamma_{a}\right)^{\alpha \beta} T_{\alpha \beta}^{\gamma}=0
$$

The space of forms admits a natural bigrading according to the degrees of the forms and their Grassmann character. The space of forms with $p$ even and $q$ odd components is denoted by $\Omega^{p, q}$; $\omega \in \Omega^{p, q}$ has the form

$$
\omega=E^{\beta_{q}} \ldots E^{\beta_{1}} E^{a_{p}} \ldots E^{a_{1}} \omega_{a_{1} \ldots a_{p} \beta_{1} \ldots \beta_{q}}
$$

The exterior derivative $d$ written out in this basis naturally involves the components of the torsion as one has to differentiate the one-form basis elements. It maps $\Omega^{p, q}$ to $\Omega^{p+1, q}+\Omega^{p, q+1}+$ $\Omega^{p-1, q+2}+\Omega^{p+2, q-1}$. Following [30] we split $d$ into its various components with respect to the bigrading. We put

$$
d=d_{0}+d_{1}+\tau_{0}+\tau_{1}
$$

where $d_{0}\left(d_{1}\right)$ is the even (odd) derivative with bidegrees $(1,0)$ and $(0,1)$ respectively, while $\tau_{0}$ and $\tau_{1}$ have bidegrees $(-1,2)$ and $(2,-1)$. These two latter operators are purely algebraic and involve the dimension-zero and dimension-three-halves components of the torsion tensor respectively. The subscripts indicate that $\tau_{0}$ is an even operator while $\tau_{1}$ is odd. Explicitly, for $\omega \in \Omega^{p, q}$, 


$$
\begin{aligned}
\left(d_{0} \omega\right)_{a_{1} \ldots a_{p+1} \beta_{1} \ldots \beta_{q}}= & \nabla_{\left[a_{1}\right.} \omega_{\left.a_{2} \ldots a_{p+1}\right] \beta_{1} \ldots \beta_{q}}+\frac{p}{2} T_{\left[a_{1} a_{2}\right.}{ }^{c} \omega_{\left.|c| a_{3} \ldots a_{p+1}\right] \beta_{1} \ldots \beta_{q}} \\
& +q(-1)^{p} T_{\left[a _ { 1 } \left(\beta_{1}\right.\right.}{ }^{\gamma} \omega_{\left.\left.a_{2} \ldots a_{p+1}\right]|\gamma| \beta_{2} \ldots \beta_{q}\right)} \\
\left(d_{1} \omega\right)_{a_{1} \ldots a_{p} \beta_{1} \ldots \beta_{q+1}}= & \left.(-1)^{p} \nabla_{\left(\beta_{1}\right.} \omega_{\left.a_{1} \ldots a_{p} \beta_{2} \ldots \beta_{q+1}\right)}+\frac{q}{2} T_{\left(\beta_{1} \beta_{2}\right.}{ }^{\gamma} \omega_{\left.\left.a_{1} \ldots a_{p}\right]|\gamma| \beta_{3} \ldots \beta_{q+1}\right)}\right) \\
& +p(-1)^{p} T_{\left(\beta _ { 1 } \left[a_{1}\right.\right.}{ }^{c} \omega_{\left.\left.|c| a_{2} \ldots a_{p}\right] \beta_{2} \ldots \beta_{q+1}\right)} \\
\left(\tau_{0} \omega\right)_{a_{1} \ldots a_{p-1} \beta_{1} \ldots \beta_{q+2}}= & \frac{p}{2} T_{\left(\beta_{1} \beta_{2}\right.}{ }^{c} \omega_{\left.|c| a_{1} \ldots a_{p-1} \beta_{3} \ldots \beta_{q+2}\right)} \\
\left(\tau_{1} \omega\right)_{a_{1} \ldots a_{p+2} \beta_{1} \ldots \beta_{q-1}}= & \frac{q}{2} T_{\left[a_{1} a_{2}\right.}{ }^{\gamma} \omega_{\left.\left.a_{3} \ldots a_{p+2}\right]|\gamma| \beta_{1} \ldots \beta_{q-1}\right)}
\end{aligned}
$$

The fact that $d^{2}=0$ implies the following identities:

$$
\begin{aligned}
\tau_{0}^{2} & =0 \\
d_{1} \tau_{0}+\tau_{0} d_{1} & =0 \\
d_{1}^{2}+d_{0} \tau_{0}+\tau_{0} d_{0} & =0 \\
d_{0} d_{1}+d_{1} d_{0}+\tau_{0} \tau_{1}+\tau_{1} \tau_{0} & =0 \\
d_{0}^{2}+d_{1} \tau_{1}+\tau_{1} d_{1} & =0 \\
d_{0} \tau_{1}+\tau_{1} d_{0} & =0 \\
\tau_{1}^{2} & =0
\end{aligned}
$$

It should be noted that, although $d^{2}=0$ is an identity by the definition of $d$, when one introduces a splitting of the tangent space into even and odd, the equations above (except for the first and last ones) are only identities provided that the torsion tensor obeys the first Bianchi identity. For example, if one applies (19) to an even one-form one finds that it is true provided that $I_{\alpha \beta \gamma}{ }^{d}=0$.

Equation (18) implies that we can consider the cohomology of $\tau_{0}$, as first noted in [30]. We set

$$
H_{\tau}^{p, q}=\left\{\omega \in \Omega^{p, q} \mid \tau_{0} \omega=0 \bmod \omega=\tau_{0} \lambda, \lambda \in \Omega^{p+1, q-2}\right\}
$$

We can now define a spinorial derivative $d_{F}$ which will act on elements of $H_{\tau}^{p, q}$. If $\omega \in[\omega] \in H_{\tau}^{p, q}$ we set

$$
d_{F}[\omega]:=\left[d_{1} \omega\right]
$$

It is easy to check that this is well-defined, i.e. $d_{1} \omega$ is $\tau_{0}$-closed, and $d_{F}[\omega]$ is independent of the choice of representative. Furthermore it is simple to check that $d_{F}^{2}=0$. This means that we can define the spinorial cohomology groups $H_{F}^{p, q}$ in the obvious fashion. 
If the dimension zero torsion tensor is flat, i.e. equal to the gamma matrix, then the cohomology groups $H_{F}^{0, q}$ are isomorphic to Berkovits's B-pure spinor cohomology groups and the spinorial cohomology groups introduced in [2]. The present definition extends this to arbitrary dimensionzero torsion and to mixed forms.

The above construction can be extended to vector-valued forms of a certain type. Let $\Omega^{p} \otimes T$ denote the space of vector-valued $p$-forms on a manifold. Any $h \in \Omega^{p} \otimes T$ defines a derivation of degree $p-1$ of the algebra of differential forms, $\Omega^{q} \ni \omega \mapsto i_{h} \omega \in \Omega^{p+q-1}$. Essentially, the vector index of $h$ is contracted with one of the indices of $\omega$ while the remaining indices of $h$ and $\omega$ are antisymmetrised. We can combine this operation with exterior differentiation to get a generalisation of the notion of Lie derivative:

$$
\mathcal{L}_{h} w:=d i_{h} \omega+(-1)^{p} i_{h} d \omega
$$

Now let $h \in \Omega^{0, p} \otimes B$, the space of $(0, p)$-forms taking their values in the even tangent space $B$, and let $\omega \in \Omega^{1,0}$. If we restrict the derivative to act in the odd direction we get an expression which is tensorial in $\omega$,

$$
d_{1} h(\omega):=d_{1} i_{h} \omega+(-1)^{p} i_{h} d_{1} \omega
$$

It is easy to check that this has the desired tensorial property that $d_{1} h(f \omega)=f d_{1} h(\omega)$. In order to define a spinorial cohomology, we have to remove the unwanted parts of $h$. We can define an action of the dimension-zero torsion in two ways, either by converting an even form index into two odd ones as for ordinary forms, or by converting an odd vector index into an even vector index and an odd form index. In components,

$$
k_{a \beta_{1} \ldots \beta_{p-2}}^{b} \mapsto T_{\left(\beta_{1} \beta_{2}\right.}^{c} k_{\left.c \beta_{3} \ldots \beta_{p}\right)}^{b}
$$

or

$$
k_{\alpha_{1} \ldots \alpha_{p-1}}^{\gamma} \mapsto k_{\left(\alpha_{1} \ldots \alpha_{p-1}\right.}^{\gamma} T_{\left.\gamma \alpha_{p}\right)}^{c}
$$

For the case that $T_{\alpha \beta}{ }^{c}$ is covariantly constant one can show that $d_{1}$ defines a derivative $d_{F}$ which squares to zero on the spaces $\Omega^{0, p} \otimes B$ modulo the two equivalences defined above. We shall refer to this as vector-valued spinorial cohomology and denote it by $\check{H}_{F}^{p}$. In the case when one has a general dimension-zero torsion one also has to factor out terms of the form $k_{\left(\alpha_{1} \ldots \alpha_{p-2}\right.}^{D} \nabla_{D} T_{\left.\alpha_{p-1} \alpha_{p}\right)}{ }^{c}$.

An application of this is to the supergravity equations of motion. We saw above that these are implied by the constraint $T_{\alpha \beta}{ }^{c}=-i\left(\gamma^{c}\right)_{\alpha \beta}$. If we now make a deformation of the supervielbein of the form $E_{A}{ }^{M} \delta E_{M}^{B}=h_{A}^{B}$ we find that the change in the dimension zero torsion is

$$
\delta T_{\alpha \beta}{ }^{c}=2 \nabla_{(\alpha} h_{\beta)}{ }^{c}+T_{\alpha \beta}{ }^{D} h_{D}{ }^{c}-2 h_{(\alpha}{ }^{D} T_{D \beta)}{ }^{c}
$$


The equations of motion will be satisfied for the deformed supervielbein if this expression vanishes. Now the essential deformation is given by $h_{\alpha}{ }^{b}$ since the other components of $h_{A}{ }^{B}$ which appear in (31) are simply field redefinitions. So the esential part of (31) is obtained by ignoring the terms of the form $T_{\alpha \beta}{ }^{d} h_{d}{ }^{c}$ and $h_{(\alpha}{ }^{\delta} T_{\delta \beta)}{ }^{c}$ which are precisely the equivalences defined in (29) and (30). Moreover, changing $h_{\alpha}{ }^{c}$ by a term of the form $k^{\gamma} T_{\gamma \alpha}{ }^{c}$ also leads to a field redefinition. Therefore we see that the possible deformations of the supervielbein which preserve the field equations are given by $\check{H}_{F}^{1}$. This space therefore tells us about on-shell degrees of freedom of the theory.

\section{Deformations in the geometrical sector}

We shall now consider deformations of the supergravity equations of motion. By this we mean we look for solutions of the Bianchi identities in a power series in some dimensionful parameter, $t$ say, with the deformations being expressed in terms of the lowest order fields. Such a solution, if consistent, will result in modified equations of motion. The lowest-order fields are themselves taken to be independent of $t$ although if we solve the equations of motion perturbatively they will depend on the parameter as well as on the solutions to the original supergravity equations in a non-local manner.

This has been discussed for $D=11$ supergravity in [21]. We begin by looking at this problem from the geometrical point of view. As observed in [2] one can look at the first-order deformations in terms of the vector-valued cohomology group $\check{H}_{F}^{2}$ (phys), where the notation indicates that in this case the coefficients are not freely given superfields but are instead functions of the physical fields. This is easily seen. The dimension one-half Bianchi identity is

$$
\nabla_{(\alpha} T_{\beta \gamma}^{d}+T_{(\alpha \beta}^{E} T_{|E| \gamma)}^{d}=0
$$

If we expand in powers of $t$ in the form $T=\stackrel{0}{T}+t \stackrel{1}{T}+\ldots$ we have at first order

$$
\nabla_{(\alpha} \stackrel{1}{T}_{\beta \gamma)}^{d}+\stackrel{0}{T}_{(\alpha \beta}^{e} \stackrel{1}{T}_{|e| \gamma)}^{d}+\stackrel{1}{T}_{(\alpha \beta}^{\epsilon} \stackrel{0}{T}_{|\epsilon| \gamma)}^{d}=0
$$

We see that the two quadratic terms here are of the form of equivalences (with respect to the lowest order dimension zero torsion) while the algebraic field redefinitions of $\stackrel{1}{\alpha \beta}^{c}$ show that this equation can be interpreted as $d_{F}\left[\tau_{0}\right]=0 .{ }^{2}$ Furthermore, one can make field redefinitions of the supervielbein which will change $\left[\tau_{0}\right]$ by the $d_{F}$ of a vector-valued one-form. Hence the deformations of the equations of motion are indeed characterised by $\check{H}_{F}^{2}$ (phys). It might be the case that there are further constraints arising at higher order, although this seems to be unlikely. We have seen that the theory can in principle be discussed in a framework where we do not need to introduce a connection or curvature; when we do, we expect to find that the geometrical Bianchi identities should serve merely to solve for the higher-dimensional components

\footnotetext{
${ }^{2}$ We use the notation $\tau_{0}$ to denote the dimension zero part of the torsion considered as a vector-valued two-form. $\stackrel{1}{\tau}_{0}$ denotes the first-order deformation of this form.
} 
of the torsion and the curvature in terms of the components of $\stackrel{1}{T}_{\alpha \beta}{ }^{c}$, these components being themselves given in terms of the basic massless supergravity field strength tensors.

From a practical point of view, however, the purely geometrical approach is not likely to be very helpful in the task of finding the explicit form of the deformation. It is expected that the first deformation will be associated with an $R^{4}$ Lagrangian, so that the parameter $t$ would be of the form $\ell^{6}$ where $\ell$ is some length scale. The analysis of the cohomology in this situation would be extremely difficult to carry out.

For this reason it seems that it may be advantageous to introduce the four-form field strength from the beginning. In particular, it is easier to analyse the question of higher-order corrections, and the cohomology groups which arise are based on ordinary differential forms and not on vector-valued ones.

\section{Four-form supergravity}

Let us now study the theory with the inclusion of a four-form field strength $G_{4}=d C$ from the beginning. The four lowest-dimensional Bianchi identities $I=d G_{4}=0 \mathrm{read}$

$$
\begin{aligned}
d_{1} G_{0,4}+\tau_{0} G_{1,3} & =0 \\
d_{0} G_{0,4}+d_{1} G_{1,3}+\tau_{0} G_{2,2} & =0 \\
d_{0} G_{1,3}+d_{1} G_{2,2}+\tau_{0} G_{3,1}+\tau_{1} G_{0,4} & =0 \\
d_{0} G_{2,2}+d_{1} G_{3,1}+\tau_{0} G_{4,0}+\tau_{1} G_{1,3} & =0
\end{aligned}
$$

We also note that $\tau_{0} G_{0,4}=0$. This is trivially true but helps to make the picture clearer from the point of view of cohomology.

Let us begin by assuming that $G_{0,4}=0$. Since

$$
G_{0,4}=d_{1} C_{0,3}+\tau_{0} C_{1,2}
$$

it is enough to take $\left[G_{0,4}\right] \in H_{\tau}^{0,4}$ to be zero. Moreover, since the gauge transformation of $C_{0,3}$ is

$$
\delta C_{0,3}=d_{1} Y_{0,2}+\tau_{0} Y_{1,1}
$$

it follows that $\left[G_{0,4}\right]=0$ is equivalent to specifying an element of $H_{F}^{0,3}$.

The $(0,5)$ component of the Bianchi identity then implies that

$$
\tau_{0} G_{1,3}=0
$$

We can examine this equation perturbatively. Since $\stackrel{0}{G}_{1,3}=0$ we only need to use the lowest-order torsion. We therefore find 


$$
\left(\gamma^{a}\right)_{(\alpha \beta} \stackrel{1}{G}{ }_{a \gamma \delta \epsilon)}=0
$$

which has the solution

$$
\stackrel{1}{G}_{a \gamma \delta \epsilon}=\left(\gamma^{b}\right)_{(\gamma \delta} K_{a b \epsilon)}+\left(\gamma_{a b}\right)_{(\gamma \delta} L_{\epsilon)}^{b}
$$

The first term on the RHS is the trivial solution since $K_{a b \gamma} \in \Omega^{2,1}$, while the second term is non-trivial except for the purely spin one-half part of $L_{\epsilon}^{b}$ which can clearly be transferred to the first term. The trivial term can be removed by a conventional constraint since $G_{1,3}=d_{0} C_{0,3}+$ $d_{1} C_{1,2}+\tau_{0} C_{2,1}$. The second term can be removed by a field redefinition of the supervielbein because

$$
\stackrel{0}{G}_{\alpha \beta c d}=-i\left(\gamma_{c d}\right)_{\alpha \beta}
$$

A (first-order) change in the supervielbein of the form $\delta E_{\alpha}=-h_{\alpha}{ }^{b} E_{b}$ will therefore give rise to a change in $G_{1,3}$ of precisely the same form as the second term in (42). As we have seen, the gamma-trace part of this change will not be required as one might expect from earlier remarks. So we can take $\stackrel{1}{G}_{1,3}=0$.

The $(1,4)$ component of the Bianchi identity then implies that

$$
\tau_{0} G_{2,2}=0
$$

Writing this out in terms of perturbations we have

$$
\left(\gamma^{b}\right)_{(\alpha \beta} \stackrel{1}{G}_{a b \gamma \delta)}+\stackrel{1}{T}_{(\alpha \beta}^{b}\left(\gamma_{a b}\right)_{\gamma \delta)}=0
$$

The only non-zero solutions to this equation can be removed by field redefinitions. In particular, one can have solutions where both tensors are scalar functions multiplied by the lowest-order constant tensors. These can be set to zero by rescaling the even and odd parts of the supervielbein separately.

At this stage we have shown that there are no non-trivial deformations of the dimension-zero torsion and hence that one would expect to recover the equations of motion of supergravity. However, this is not quite right as we are using a Lorentzian formalism and we have already used up the scale transformation to deal with the dimension-zero component of $G$. We therefore have to check at dimension one-half. Given that $I_{0,5}=I_{1,4}=0$ and that $d I=0$ we find that the information contained in $I_{2,3}$ satisfies $\tau_{0} I_{2,3}=0$. This equation is solved by

$$
I_{a b \gamma \delta \epsilon}=\left(\gamma^{c}\right)_{(\gamma \delta} J_{a b c \epsilon)}+\left(\gamma_{a b}\right)_{(\gamma \delta} K_{\epsilon)}
$$

where the first term is $\tau_{0}$ trivial and the second is not; indeed, the spin-half term in $J$ does not have the same gamma-matrix structure as $K$. The equation itself is 


$$
d_{1} \stackrel{0}{G}_{2,2}+\tau_{0} \stackrel{1}{G}_{3,1}=0
$$

Note that the first term is non-zero because $d_{1}$ contains the dimension one-half torsion. There is a spin-half field in the first term (contained in the dimension one-half torsion) and a spin-onehalf field in $\stackrel{1}{G}_{3,1}$; but, as we have just seen, there are two independent constraints on these fields so that both of them must vanish. It follows from (46) that the remainder of ${ }_{G 3,1}^{1}$ vanishes too.

We therefore conclude that any deformation of the supergravity equations of motion for which $\left[G_{0,4}\right]=0$ is trivial. Having established this to first order we can iterate the procedure. In this perturbative sense, therefore, we can say that the equations of motion of supergravity are determined by an element of $H_{F}^{0,3}$. This seems to depend on a choice of an odd tangent bundle, but it then turns out that this choice must be such that the dimension-zero torsion is trivial.

\section{Deformations in the four-form theory}

We now go on to consider the deformations of the supergravity equations which arise when $\left[G_{0,4}\right] \neq 0$. The lowest-dimension Bianchi identity $I_{0,5}$ reads

$$
d_{1} G_{0,4}+\tau_{0} G_{1,3}=0
$$

Clearly this implies that $\left[d_{1} G_{0,4}\right]=0$ thereby determining an element of the cohomology group $H_{F}^{0,4}$. For a physical deformation of the theory we require $G_{0,4}$ to be a function of the supergravity fields, so we are really interested in elements of $H_{F}^{0,4}$ (phys). We claim that, given such an element, the rest of the Bianchi identities determine the other components of $G_{4}$ in terms of derivatives of $G_{0,4}$ up to field redefinitions and gauge transformations. In addition, the dimension-zero torsion is determined, i.e the tensors $X$ and $Y$ at first order, as well as the dimension one-half spinor which vanishes in the undeformed theory but which will be a function of known fields in the deformed case. We shall give a brief outline of this below and a more detailed account in terms of the representations involved in section 6 .

Perhaps the easiest way to see the result is to examine the equation $d I_{5}=0$ for the components of the Bianchi identity. If $I_{0,5}$ is satisfied then the information in $I_{1,4}$ will be subject to the constraint $\tau_{0} I_{1,4}=0$ and so on, provided that the geometrical Bianchi identities are satisfied. However, from dimension one-half onwards we need to take this into account too. In this way we get a sequence of algebraic equations whose solution will tell us the information contained in each $I_{p . q}$ given that the lower-dimensional ones have been solved.

Assuming that $I_{0,5}=0$ we find $\tau_{0} I_{1,4}=0$ which in components is solved by

$$
I_{a \beta \gamma \delta \epsilon}=\left(\gamma^{b}\right)_{(\beta \gamma} J_{a b \delta \epsilon)}+\left(\gamma_{a b}\right)_{(\beta \gamma} K_{\delta \epsilon)}^{b}
$$

¿From this we can deduce that $I_{1,4}$ allows us to solve for the dimension-zero torsion and $G_{4}$ deformations up to field redefinitions as in the previous case. To go from $I_{1,4}$ to $I_{2,3}$ we have to take into account the fact that $G_{2,2}$ has a non-vanishing component at zeroth order, so that we 
are not allowed to use the equation $d_{1} \tau_{0}+\tau_{0} d_{1}=0$ immediately. One finds, by differentiating $I_{1,4}$ and then setting the result to zero that

$$
\left(d_{1} \tau_{0}+\tau_{0} d_{1}\right) G_{2,2}=\tau_{0} I_{2,3}
$$

or, in indices, and remembering that the Bianchi identities are satisfied to zeroth order,

$$
I_{(\alpha \beta \gamma}^{b}\left(\gamma_{a b}\right)_{\delta \epsilon)}=\left(\gamma^{b}\right)_{(\alpha \beta} I_{a b \gamma \delta \epsilon)}
$$

This equation is solved by

$$
I_{a b \gamma \delta \epsilon}=\left(\gamma^{c}\right)_{(\gamma \delta} J_{a b c \epsilon)}+\left(\gamma_{a b}\right)_{(\gamma \delta} K_{\epsilon)}+2\left(\gamma_{c[a}\right)_{(\gamma \delta} L_{\epsilon) b}^{c}-M_{(\gamma \delta}{ }^{\eta}\left(\gamma_{a b}\right)_{\epsilon) \eta}
$$

and

$$
I_{\alpha \beta \gamma}{ }^{b}=\left(\gamma^{c}\right)_{(\alpha \beta} L_{\gamma) c}^{b}+M_{(\alpha \beta}{ }^{\eta}\left(\gamma^{b}\right)_{\gamma) \eta}
$$

Furthermore, the components of $L$ and $M$ which correspond to field redefinitions of the dimension one-half connection and the choice of even tangent bundle cancel out in these expressions. Setting $L$ and $M$ equal to zero therefore determines the dimension one-half torsion, up to these field redefinitions, while setting $J=0$ determines the dimension one-half component of $G_{4}$. Finally, if we put $K=0$ we determine the dimension one-half Weyl fermion as an explicit function of the fields at first order. The remainder of the dimension one-half geometrical Bianchi identity must be automatically satisfied by the deformed dimension zero torsion determined from $G_{0,4}$ up to equivalences of the type given in (29) and (30), and this therefore implies that the equation $d_{F}\left[\tau_{0}^{1}\right]=0$ discussed earlier is satisfied.

We can continue this analysis at the next order where we encounter the dimension one geometrical Bianchi identities. However, it is likely that they are automatically satisfied if the dimension one-half one is and so, at this stage, we can assume that the d-algebra is satisfied. We then find that $\tau_{0} I_{3,2}=0$ which allows us to relate $G_{4,0}$ to the dimension-one torsion. To summarise this section, we have shown that the first-order deformation of the theory is completely determined by an element of $H_{F}^{0,4}$ (phys) given which one can compute the first-order deformations of all of the remaining components of the four-form field strength as well as the components of the torsion and curvature in a systematic way using the Bianchi identities.

\section{Seven-form supergravity}

It is well-known that one can introduce a seven-form field strength $G_{7}$ as well as the four-form which we shall now denote by $G_{4}$. In supergravity itself it obeys the Bianchi identity

$$
d G_{7}=\frac{1}{2} G_{4}^{2}
$$


The only non-vanishing components of $G_{7}$ are $G_{7,0}$ which is the dual of $G_{4,0}$ and $G_{5,2}$ which is given by

$$
G_{a b c d e \alpha \beta}=-i\left(\gamma_{a b c d e}\right)_{\alpha \beta}
$$

This formulation was first discussed in superspace in [29].

In the deformed theory, however, we have to take into account the Chern-Simons term which is necessary in order to cancel the anomaly on the fivebrane [31]. The modified Bianchi identity is

$$
d G_{7}=\frac{1}{2} G_{4}^{2}+\beta X_{8}
$$

where $\beta$ is a parameter of dimension $\ell^{6}$ and $X_{8}=\operatorname{tr}\left(R^{4}\right)-\frac{1}{4}\left(\operatorname{tr}\left(R^{2}\right)\right)^{2}$. The necessity of including $X_{8}$ in the superspace Bianchi identity was observed in [21], although it should be noted that $G_{7}$ is not the superspace dual of $G_{4}$, and neither is $G_{7,0}$ the dual of $G_{4,0}$ in the presence of higher-order corrections. In the general (56) will determine $G_{7,0}$ to be the dual of $G_{4,0}$ plus many other correction terms with one or more powers of $\beta$.

Provided that this equation is consistent, so that one can find a particular solution corresponding to the $X$-term (which can be constructed from the zeroth order supergravity fields), the question of uniqueness then boils down to solving the original identity, (54), at first order in $\beta$. If $G_{0,7} \neq 0$, then it defines an element of $H_{F}^{0,7}(p h y s)$. If this group is trivial (at this dimension) then we must consider $G_{1,6}$ which will satisfy $\tau_{0} G_{1,6}=0$ in this case. However, the group $H_{\tau}^{1,6}$ is not trivial, and this will lead to an element of the spinorial cohomology group $H_{F}^{1,6}($ phys $)$. If this is trivial as well at this dimension, we shall have $\tau_{0} G_{2,5}=0$. The cohomology group $H_{\tau}^{2,5}$ is also non-trivial and so we get an element of $H_{F}^{2,5}$ (phys). If this element should also vanish, the next Bianchi, at dimension -1 involves the $G_{4}$ field, and reads, given that $G_{0,7}=G_{1,6}=G_{2,5}=0$,

$$
\stackrel{0}{\tau}_{0} \stackrel{1}{G}_{3,4}=\stackrel{0}{G_{2,2}} \stackrel{1}{G}_{0,4}
$$

In index notation,

$$
\left(\gamma^{c}\right)_{(\alpha \beta} \stackrel{1}{G}_{a b c \gamma \delta \epsilon \eta)}=\left(\gamma_{a b}\right)_{(\alpha \beta} \stackrel{1}{G}_{\gamma \delta \epsilon \eta)}
$$

This equation admits a trivial solution for $\stackrel{1}{G}_{3,4}$ and the $\tau_{0}$-trivial expression for $\stackrel{1}{G}_{0,4}$ can also easily be seen to be a solution for a simple choice of the left-hand side. But there are no other solutions, and this implies that both $G_{3,4}$ and $G_{0,4}$ are unchanged at first order. To see this one can multiply (58) by six factors of a B-pure spinor $u$. The left-hand side is annihilated due to the $\gamma^{c}$ factor, but the right-hand side is not. Indeed, only the $\tau_{0}$-trivial representation in $G_{0,4}$ is killed. There are three non-trivial representations in $G_{0,4}$ which are $(n, k)$-tensors of the form $(2,2),(2,5)$ and $(5,5)$. None of these is annihilated so that $(58)$ indeed implies that $G_{0,4}$ is $\tau_{0}$-trivial. The triviality of $G_{3,4}$ then folllows from the fact that $H_{\tau}^{3,4}=0$. From the earlier discussion of the $G_{4}$ Bianchi identity, it is clear, at this stage, that we recover the standard zeroth order theory. 
This analysis shows that the possible ambiguities in solving the Bianchi identities at order $\beta$

are described by the cohomology groups $H_{F}^{0,7}$ (phys), $H_{F}^{1,6}($ phys $)$ and $H_{F}^{2,5}$ (phys). Moreover, a solution of the modified Bianchi identity will automatically determine the first-order correction to $G_{0,4}$ and hence the modified equations of motion. Therefore, we conclude that, if these three cohomology groups are zero at first order in $\beta$, the complete equations of motion and supersymmetry transformations can be determined systematically by solving the Bianchi identities.

We shall now argue that it is indeed the case that these groups vanish at this order, if we assume that the invariants we should consider should be at least quartic in the fields. In fact, if this assumption is made, there is nothing one can write down for $G_{0,7}$ of the right dimension. However, for $G_{1,6}$ one could have terms of the form $W^{4}$, while for $G_{2,5}$ we could have terms of the form $W^{3} \Psi$ where $\Psi$ is the gravitino field strength. We shall argue below by an indirect argument that this is not possible, and so we conclude that we can write $X_{0,8}$ in the form $\tau_{0} G_{1,6}$ and that this solves for $G_{1,6}$ up to field redefinitions.

\section{Action principle}

One way of going about the construction of integral super-invariants in $D$ dimensions is to consider a closed $D$-form $L_{D}$, say, up to exact terms which would modify the integrand by a total derivative $[32,33]$. The invariant is given by

$$
S=\int d^{D} x \epsilon^{m_{1} \ldots m_{D}} L_{m_{1} \ldots m_{D}} \mid
$$

where the bar denotes the evaluation of a superfield at $\theta=0$.

This construction yields an expression which is automatically invariant under local supersymmetry transformations and spacetime diffeomorphisms. Under a infinitesimal diffeomorphism in superspace one has

$$
\delta L_{D}=\mathcal{L}_{v} L_{D}=d i_{v} L_{D}+i_{v} d L_{D}=d i_{v} L_{D}
$$

from which one reads that the integrand above transforms as a total derivative under the spacetime transformations corresponding to zeroth order terms in the superspace vector field $v$. These are just local supersymmetry transformations and spacetime diffeomorphisms.

There is a class of actions, namely those terms of the Chern-Simons type, which can be usefully discussed using this method. In this case there is a closed $D+1$-form, $W_{D+1}$, which can be written in two ways: as the exterior derivative of the potential $Z_{D}$, the Chern-Simons term, which defines $W$ or as the exterior derivative of a well-defined $D$-form $K_{D}$, say. The difference between the two then automatically gives a closed Lagrangian form $L_{D}=K_{D}-Z_{D}$. The potential term obviously contains the Chern-Simons term in the Lagrangian. This method can be used to derive in a systematic way the Green-Schwarz actions for branes in the superembedding formalism starting from the Wess-Zumino term. [34, 35].

In the present case, the twelve-form we need to consider is 


$$
W_{12}=\frac{1}{2} G_{4}^{3}+3 \beta G_{4} X_{8}
$$

In fact, this form should lead to the sum of the zeroth- and first-order actions provided one exercises sufficient care with the use of an on-shell formalism.

Now suppose this does not give a unique invariant of $R^{4}$ type. In that case we would expect that there should be a closed 11-form, $L_{11}$. However, we claim that the cohomology class of such a form must be trivial to order $\beta$ provided that we restrict ourselves to terms which are at least quartic in the fields. To see this we note that the dimension of $L_{11,0}$ is 8 and since $W$ has dimension 1 , the lowest-dimensional object available is $W^{4}$ at dimension 4 . This corresponds to the component $L_{3,8}$ and so we must infer that $L_{2,9}=L_{1,10}=L_{0,11}=0$. But then we must have $\tau_{0} L_{3,8}=0$. However, it seems likely that all of the $\tau$-cohomology groups $H_{\tau}^{p, q}$ vanish for $p \geq 3$. This can be supported by the principle of maximal propagation of representations presented in [2], but one should be aware that this does not give an unambiguous result. On the other hand, all of the known non-trivial examples of $\tau$-cohomology involve a factor of $\gamma_{a b}$ and make use of the membrane identity

$$
\left(\gamma^{a}\right)_{(\alpha \beta}\left(\gamma_{a b}\right)_{\gamma \delta)}=0
$$

When $p \geq 3$ the putative cocycle must involve another index and there is no obvious way of doing this without introducing a second factor of $\gamma_{2}$. However, one then runs into the five-brane identity

$$
\left(\gamma^{a}\right)_{(\alpha \beta}\left(\gamma_{a b c d e}\right)_{\gamma \delta)}=\left(\gamma_{[a b}\right)_{(\alpha \beta}\left(\gamma_{c d]}\right)_{\gamma \delta)}
$$

which implies a cocycle with two $\gamma_{2} \mathrm{~s}$ is in fact a coboundary. Assuming this to be correct, we conclude that any solution to $d L_{11}=0$ at $\ell^{6}$ which is at least quartic in the fields must be trivial.

\section{The $G_{4}$ Bianchi identities}

In this section we give a more detailed discussion of the four-form Bianchi identity at dimension $-\frac{1}{2}$ and we derive a number of constraints on $G_{0,4}$. We then proceed to dimension zero to give the expression of the zero-dimension torsion in terms of (derivatives of) the superfields in $G_{0,4}$. We have found the programs LiE [36] and Gamma [37] useful for representation-theoretic considerations and Gamma-matrix computations respectively. An explanation of the grouptheoretic notation is given in appendix $\mathrm{C}$.

To zeroth order the only nonzero components of $G$ are

$$
G_{a b c d}\left(=W_{a b c d}\right) ; \quad G_{a b \alpha \beta}=-i\left(\gamma_{a b}\right)_{\alpha \beta}
$$

In components the $\mathrm{BI}$ reads,

$$
\nabla_{\left[A_{1}\right.} G_{\left.A_{2} \ldots A_{5}\right\}}+2 T_{\left[A_{1} A_{2} \mid\right.}{ }^{F} G_{\left.F \mid A_{3} A_{4} A_{5}\right\}}=0
$$


The invariance $G \rightarrow G+d C$ reads in components,

$$
\delta G_{A_{1} \ldots A_{4}}=\frac{3}{2} T_{\left[A_{1} A_{2} \mid\right.}^{F} C_{\left.F \mid A_{3} A_{4}\right\}}+\nabla_{\left[A_{1}\right.} C_{\left.A_{2} A_{3} A_{4}\right\}} .
$$

In addition we have the freedom of redefinitions of the vielbein $E_{A}{ }^{M} \delta E_{M}{ }^{B}:=h_{A}{ }^{B}$. These induce shifts in $G$ according to,

$$
\delta G_{A_{1} \ldots A_{4}}=-4 h_{\left[A_{1} \mid\right.}^{F} G_{\left.F \mid A_{2} A_{3} A_{4}\right\}}
$$

Note that we are not free to vary all the components of $E_{M}{ }^{A}$, because some of them have already been used to remove some components of the torsion. The only components of the vielbein which are still free to use in redefinitions of the type above, are [21],

$$
\left.h_{\alpha}{ }^{a}\right|_{(00001)} ;\left.\quad h_{\alpha}{ }^{a}\right|_{(10001)} ;\left.\quad h_{\alpha}{ }^{\beta}\right|_{(00000)} ;\left.\quad h_{\alpha}{ }^{\beta}\right|_{(01000)}
$$

\section{The BI at dimension $-\frac{1}{2}$.}

To first order the dimension $-\frac{1}{2}$ Bianchi identity reads,

$$
0=\nabla_{\left(\alpha_{1}\right.} G_{\left.\alpha_{2} \ldots \alpha_{5}\right)}-2 i\left(\gamma^{f}\right)_{\left(\alpha_{1} \alpha_{2} \mid\right.} G_{\left.f \mid \alpha_{3} \alpha_{4} \alpha_{5}\right)} .
$$

In order to solve it we need the expansions, in irreducible representations, of the fields $G_{\alpha_{1} \ldots \alpha_{4}}$, $\nabla_{\alpha_{1}} G_{\alpha_{2} \ldots \alpha_{5}}$ and $G_{a \alpha_{1} \alpha_{2} \alpha_{3}}$. The field $G_{\alpha_{1} \ldots \alpha_{4}}$ decomposes as,

$$
\begin{gathered}
\otimes_{s}^{4}(00001) \sim(00002) \oplus(00010) \oplus(00100) \oplus(00000) \oplus(00004) \oplus(01002) \\
\oplus(10002) \oplus(02000) \oplus(11000) \oplus(20000)
\end{gathered}
$$

Redefinitions $\delta G_{\alpha_{1} \ldots \alpha_{4}}$ of the type (66) vanish at this order. Redefinition (65) reads

$$
\delta G_{\alpha_{1} \ldots \alpha_{4}}=-\frac{3 i}{2}\left(\gamma^{f}\right)_{\left(\alpha_{1} \alpha_{2} \mid\right.} C_{\left.f \mid \alpha_{3} \alpha_{4}\right)}+\nabla_{\left(\alpha_{1}\right.} C_{\left.\alpha_{2} \alpha_{3} \alpha_{4}\right)}
$$

The superfield $C_{a \alpha_{1} \alpha_{2}}$ decomposes as

$$
\begin{aligned}
& \otimes_{s}^{2}(00001) \otimes(10000) \sim(00002) \oplus(00010) \oplus(00100) \oplus(01000) \oplus(10000) \\
& \oplus(00000) \oplus(10002) \oplus(11000) \oplus(20000)
\end{aligned}
$$

Therefore, we can use all but the (01000) and (10000) irreps in $C_{a \alpha_{1} \alpha_{2}}$ to fix,

$$
G_{\alpha_{1} \ldots \alpha_{4}} \sim \oplus(00004) \oplus(01002) \oplus(02000)
$$

The superfield $C_{\alpha_{1} \alpha_{2} \alpha_{3}}$ will not be used at this stage. Explicitly we expand,

$$
\begin{aligned}
G_{\alpha_{1} \ldots \alpha_{4}} & =\frac{1}{8}\left(\gamma^{a_{1} a_{2}}\right)_{\left(\alpha_{1} \alpha_{2}\right.}\left(\gamma^{b_{1} b_{2}}\right)_{\left.\alpha_{3} \alpha_{4}\right)} A_{a_{1} a_{2} ; b_{1} b_{2}} \\
& +\frac{1}{240}\left(\gamma^{a_{1} \ldots a_{5}}\right)_{\left(\alpha_{1} \alpha_{2}\right.}\left(\gamma^{b_{1} b_{2}}\right)_{\left.\alpha_{3} \alpha_{4}\right)} B_{a_{1} \ldots a_{5} ; b_{1} b_{2}} \\
& +\frac{1}{28800}\left(\gamma^{a_{1} \ldots a_{5}}\right)_{\left(\alpha_{1} \alpha_{2}\right.}\left(\gamma^{b_{1} \ldots b_{5}}\right)_{\left.\alpha_{3} \alpha_{4}\right)} C_{a_{1} \ldots a_{5} ; b_{1} \ldots b_{5}}
\end{aligned}
$$


The field $G_{\alpha_{1} \ldots \alpha_{4}}$ enters the BI through its supercovariant derivative, so we also need to expand to level $\theta^{1}$ in the fields $A, B, C$.

$D A_{a_{1} a_{2} ; b_{1} b_{2}}:$

$$
(00001) \otimes(02000) \sim(01001) \oplus(11001) \oplus(02001)
$$

Explicitly $^{3}$ :

$$
\begin{aligned}
D A_{a_{1} a_{2} ; b_{1} b_{2}} & =\hat{\Pi}^{(2,2)}\left(A_{a_{1} a_{2} ; b_{1} b_{2}}^{1}\right) \\
& +\hat{\Pi}^{(2,2)}\left(\gamma_{b_{1}} A_{a_{1} a_{2} ; b_{2}}^{1}\right) \\
& +\hat{\Pi}^{(2,2)}\left(\gamma_{b_{1} b_{2}} A_{a_{1} a_{2}}^{1}\right)
\end{aligned}
$$

$D B_{a_{1} \ldots a_{5} ; b_{1} b_{2}}:$

$$
\begin{aligned}
(00001) \otimes(01002) \sim & (00003) \oplus(00011) \oplus(00101) \oplus(01001) \\
& \oplus(10003) \oplus(11001) \oplus(01003) \oplus(02001) \oplus \ldots
\end{aligned}
$$

where the ellipses stand for the irreps that do not enter the BI. Explicitly:

$$
\begin{aligned}
D B_{a_{1} \ldots a_{5} ; b_{1} b_{2}} & =\hat{\Pi}^{(5,2)}\left(\gamma_{b_{1} b_{2}} B_{a_{1} \ldots a_{5}}^{1}\right) \\
& +\hat{\Pi}^{(5,2)}\left(\gamma_{b_{1} b_{2} a_{1}} B_{a_{2} \ldots a_{5}}^{1}\right) \\
& +\hat{\Pi}^{(5,2)}\left(\gamma_{b_{1} b_{2} a_{1} a_{2}} B_{a_{3} a_{4} a_{5}}^{1}\right) \\
& +\hat{\Pi}^{(5,2)}\left(\gamma_{b_{1} b_{2} a_{1} a_{2} a_{3}} B_{a_{4} a_{5}}^{1}\right) \\
& +\hat{\Pi}^{(5,2)}\left(B_{a_{1} \ldots a_{5} ; b_{1} b_{2}}^{1}\right) \\
& +\hat{\Pi}^{(5,2)}\left(\gamma_{b_{1}} B_{a_{1} \ldots a_{5} ; b_{2}}^{1}\right) \\
& +\hat{\Pi}^{(5,2)}\left(\gamma_{a_{1} a_{2} a_{3}} B_{a_{4} a_{5} ; b_{1} b_{2}}^{1}\right) \\
& +\hat{\Pi}^{(5,2)}\left(\gamma_{b_{1} a_{1} a_{2} a_{3}} B_{a_{4} a_{5} ; b_{2}}^{1}\right) \\
& +\ldots
\end{aligned}
$$

$D C_{a_{1} \ldots a_{5} ; b_{1} \ldots b_{5}}:$

$$
(00001) \otimes(00004) \sim(00003) \oplus(10003) \oplus(01003) \oplus(00005) \oplus \ldots
$$

Explicitly:

$$
\begin{aligned}
D C_{a_{1} \ldots a_{5} ; b_{1} \ldots b_{5}} & =\hat{\Pi}^{(5,5)}\left(\gamma_{a_{1} \ldots a_{5}} C_{b_{1} \ldots b_{5}}^{1}\right) \\
& +\hat{\Pi}^{(5,5)}\left(C_{a_{1} \ldots a_{5} ; b_{1} \ldots b_{5}}^{1}\right) \\
& +\hat{\Pi}^{(5,5)}\left(\gamma_{b_{1} b_{2} b_{3}} C_{a_{1} \ldots a_{5} ; b_{4} b_{5}}^{1}\right) \\
& +\hat{\Pi}^{(5,5)}\left(\gamma_{b_{1} \ldots b_{4}} C_{a_{1} \ldots a_{5} ; b_{5}}^{1}\right) \\
& +\ldots
\end{aligned}
$$

\footnotetext{
${ }^{3}$ To simplify the notation we will omit the antisymmetrization brackets. Antisymmetrization is understood in the $\left(a_{1} a_{2}\right)$ and $\left(b_{1}, b_{2}\right)$ indices. Similarly in $(72,73)$ below. A more detailed discussion of the projections $\hat{\Pi}^{(p, q)}$ onto the irreducible part of a $(p, q)$-tensor can be found in appendix A. The explicit form of the expansions (71-73) is given in appendix $\mathrm{B}$.
} 
The field $G_{a \alpha_{1} \alpha_{2} \alpha_{3}}$ decomposes as,

$$
\begin{aligned}
\otimes_{s}^{3}(00001) \otimes(10000) \sim & (00003) \oplus(00011) \oplus(00101) \oplus 2(01001) \\
& \oplus 3(10001) \oplus 2(00001) \oplus(10003) \oplus(11001) \oplus(20001)
\end{aligned}
$$

Redefinition (65) reads

$$
\begin{aligned}
\delta G_{a \alpha_{1} \alpha_{2} \alpha_{3}} & =\frac{3}{4} T_{a\left(\alpha_{1} \mid\right.}{ }^{\beta} C_{\left.\beta \mid \alpha_{2} \alpha_{3}\right)}+\frac{1}{4} D_{a} C_{\alpha_{1} \alpha_{2} \alpha_{3}}-\frac{3}{4} D_{\left(\alpha_{1} \mid\right.} C_{\left.a \mid \alpha_{2} \alpha_{3}\right)} \\
& -\frac{3 i}{4}\left(\gamma^{f}\right)_{\left(\alpha_{1} \alpha_{2} \mid\right.} C_{\left.f a \mid \alpha_{3}\right)}
\end{aligned}
$$

The field $C_{a_{1} a_{2} \alpha}$ decomposes as,

$$
(01000) \otimes(00001) \sim(01001) \oplus(10001) \oplus(00001)
$$

The irreps on the rhs above can be used to eliminate the corresponding irreps in the decomposition of $G_{a \alpha_{1} \alpha_{2} \alpha_{3}}$. The fields $C_{\alpha_{1} \alpha_{2} \alpha_{3}}$ and $C_{a \alpha_{1} \alpha_{2}}$ will not be used at this stage. Redefinitions $\delta G_{a \alpha_{1} \alpha_{2} \alpha_{3}}$ of the type (66) read,

$$
\begin{aligned}
\delta G_{a \alpha_{1} \alpha_{2} \alpha_{3}} & =3 h_{\left(\alpha_{1} \mid\right.}{ }^{f} G_{\left.f a \mid \alpha_{2} \alpha_{3}\right)} \\
& =3 i\left(\gamma_{a}{ }^{m}\right)_{\left(\alpha_{1} \alpha_{2} \mid\right.} V_{\left.m \mid \alpha_{3}\right)}+3 i\left(\gamma_{a}{ }^{m}\right)_{\left(\alpha_{1} \alpha_{2} \mid\right.}\left(\gamma_{m} V\right)_{\left.\mid \alpha_{3}\right)}
\end{aligned}
$$

where we have decomposed the vielbein variation as

$$
h_{\alpha}^{a}=\left(\gamma^{a}\right)_{\alpha}^{\beta} V_{\beta}+V_{\alpha}^{a}
$$

into a vector-spinor $V_{a \alpha}$ and a spinor $V_{\alpha}$ part. The vector-spinor part of the vielbein can be used to eliminate one of the two remaining vector-spinors in the decomposition of $G_{a \alpha_{1} \alpha_{2} \alpha_{3}}$. Naively one might expect that the spinor part of the vielbein could also be used in order to eliminate the remaining spinor in $G_{a \alpha_{1} \alpha_{2} \alpha_{3}}$. However this is not the case. The reason is that the spinor part of the vielbein induces a redefinition (cf (76) above) of the same form as the redefinition induced by the spinor part of $C_{a b \alpha}$,

$$
\left.\delta G_{a \alpha_{1} \alpha_{2} \alpha_{3}}\right|_{(00001)} \sim\left(\gamma^{m}\right)_{\left(\alpha_{1} \alpha_{2} \mid\right.}\left(\gamma_{m a} C\right)_{\left.\mid \alpha_{3}\right)}
$$

as can be seen using the identity

$$
\left(\gamma^{m}\right)_{\left(\alpha_{1} \alpha_{2} \mid\right.}\left(\gamma_{m a}\right)_{\left.\mid \alpha_{3}\right)}^{\alpha}=-\left(\gamma_{a}^{m}\right)_{\left(\alpha_{1} \alpha_{2} \mid\right.}\left(\gamma_{m}\right)_{\left.\mid \alpha_{3}\right)}{ }^{\alpha}
$$

To conclude, after taking the field redefinitions above into account, the expansion of $G_{a \alpha_{1} \alpha_{2} \alpha_{3}}$ reads,

$$
\begin{aligned}
G_{a \alpha_{1} \alpha_{2} \alpha_{3}} \sim(00003) \oplus(00011) \oplus(00101) \oplus(01001) \\
\\
\oplus(10001) \oplus(00001) \oplus(10003) \oplus(11001) \oplus(20001)
\end{aligned}
$$


We therefore arrive at the following explicit expansion,

$$
\begin{aligned}
i G_{a \alpha_{1} \alpha_{2} \alpha_{3}} & =\frac{1}{120}\left(\gamma_{a}^{i_{1} \ldots i_{5}}\right)_{\left(\alpha_{1} \alpha_{2} \mid\right.}\left(T_{i_{1} \ldots i_{5}}\right)_{\left.\mid \alpha_{3}\right)} \\
& +\frac{1}{24}\left(\gamma_{a}^{i_{1} \ldots i_{4}}\right)_{\left(\alpha_{1} \alpha_{2} \mid\right.}\left(T_{i_{1} \ldots i_{4}}\right)_{\left.\mid \alpha_{3}\right)} \\
& +\frac{1}{6}\left(\gamma^{i_{1} i_{2}}\right)_{\left(\alpha_{1} \alpha_{2} \mid\right.}\left(T_{a i_{1} i_{2}}\right)_{\left.\mid \alpha_{3}\right)} \\
& +\frac{1}{2}\left(\gamma^{i_{1} i_{2}}\right)_{\left(\alpha_{1} \alpha_{2} \mid\right.}\left(\gamma_{a} T_{i_{1} i_{2}}\right)_{\left.\mid \alpha_{3}\right)} \\
& +\left(\gamma^{i}\right)_{\left(\alpha_{1} \alpha_{2} \mid\right.}\left(\gamma_{i} T_{a}\right)_{\left.\mid \alpha_{3}\right)} \\
& +\left(\gamma_{a}\right)_{\left(\alpha_{1} \alpha_{2} \mid\right.}(T)_{\left.\mid \alpha_{3}\right)} \\
& +\frac{1}{120}\left(\gamma^{i_{1} \ldots i_{5}}\right)_{\left(\alpha_{1} \alpha_{2} \mid\right.}\left(T_{i_{1} \ldots i_{5} ; a}\right)_{\left.\mid \alpha_{3}\right)} \\
& +\frac{1}{2}\left(\gamma^{i_{1} i_{2}}\right)_{\left(\alpha_{1} \alpha_{2} \mid\right.}\left(T_{i_{1} i_{2} ; a}\right)_{\left.\mid \alpha_{3}\right)} \\
& +\left(\gamma^{i}\right)_{\left(\alpha_{1} \alpha_{2} \mid\right.}\left(T_{i ; a}\right)_{\left.\mid \alpha_{3}\right)}
\end{aligned}
$$

The following table summarizes the various contributions to the BI for each irrep:

\begin{tabular}{|c||c|c|c|c|}
\hline B.I: & $G_{a \alpha_{1} \alpha_{2} \alpha_{3}}:$ & $D C_{a_{1} \ldots a_{5} ; b_{1} \ldots b_{5}}:$ & $D B_{a_{1} \ldots a_{5} ; b_{1} b_{2}}:$ & $D A_{a_{1} a_{2} ; b_{1} b_{2}}:$ \\
\hline \hline$(00001)$ & 1 & - & - & - \\
\hline$(00003)$ & 1 & 1 & 1 & - \\
\hline$(00005)$ & - & 1 & - & - \\
\hline$(00011)$ & 1 & - & 1 & - \\
\hline$(00101)$ & 1 & - & 1 & - \\
\hline$(01001)$ & 1 & - & 1 & 1 \\
\hline$(01003)$ & - & 1 & - & - \\
\hline$(02001)$ & - & - & 1 & - \\
\hline$(10001)$ & 1 & - & 1 & 1 \\
\hline$(10003)$ & 1 & 1 & - & - \\
\hline$(11001)$ & 1 & - & & \\
\hline$(20001)$ & 1 & - & & 1 \\
\hline
\end{tabular}

By inspection of the table above, we can guess that the following will happen: a) The BI projected onto the irreducible representations (00005), (01003) and (02001), will give three constraints on the superfield $G_{\alpha_{1} \ldots \alpha_{4}}$. b) The (00001), (10001) and (20001) components of $G_{a \alpha_{1} \alpha_{2} \alpha_{3}}$ will be set to zero by the BI. c) The remaining irreps of the BI will be used to express the rest of the components of $G_{a \alpha_{1} \alpha_{2} \alpha_{3}}$ as linear combinations of the components of $D G_{\alpha_{1} \ldots \alpha_{4}}$. Getting the precise relations requires some tedious calculations. Our strategy will be to plug in the BI the field expansions given above and to solve by projecting onto each irreducible representation using the formulae in appendices $\mathrm{A}$. and $\mathrm{B}$.

The solution to the dimension $-\frac{1}{2}$ BI reads, 


$$
\begin{aligned}
T_{a_{1} \ldots a_{5}} & =-\frac{11}{4} C_{a_{1} \ldots a_{5}}^{1}-2 B_{a_{1} \ldots a_{5}}^{1} \\
T_{a_{1} \ldots a_{4}} & =-\frac{26}{25} B_{a_{1} \ldots a_{4}}^{1} \\
T_{a_{1} a_{2} a_{3}} & =\frac{351}{175} B_{a_{1} a_{2} a_{3}}^{1} \\
T_{a_{1} a_{2}} & =-\frac{9}{35} B_{a_{1} a_{2}}^{1}-A_{a_{1} a_{2}}^{1} \\
T_{a} & =0 \\
T & =0 \\
T_{a_{1} \ldots a_{5} ; b} & =\frac{3}{5} C_{a_{1} \ldots a_{5} ; b}^{1}+\frac{1}{5} B_{a_{1} \ldots a_{5} ; b}^{1} \\
T_{a_{1} a_{2} ; b} & =-\frac{63}{200} B_{a_{1} a_{2} ; b}^{1}+\frac{1}{8} A_{a_{1} a_{2} ; b}^{1} \\
T_{a ; b} & =0 .
\end{aligned}
$$

The equations above are equivalent to the statement that the superfields in $G_{1,3}$ are expressible in terms of the superfields in $G_{0,4}$. In addition we have three constraints,

$$
\begin{aligned}
& 0=C_{a_{1} \ldots a_{5} ; b_{1} \ldots b_{5}}^{1} \\
& 0=B_{a_{1} \ldots a_{5} ; b_{1} b_{2}}^{1}-\frac{3}{10} C_{a_{1} \ldots a_{5} ; b_{1} b_{2}}^{1} \\
& 0=A_{a_{1} a_{2} ; b_{1} b_{2}}^{1}-\frac{7}{5} B_{a_{1} a_{2} ; b_{1} b_{2}}^{1} .
\end{aligned}
$$

\section{The BI at dimension 0 .}

In order to solve for the dimension-zero torsion, it suffices to focus on the (11000) and (10002) irreps, since these are the ones that parametrise the deformations of ordinary supergravity. Let us expand

$$
T_{\alpha \beta}^{a}=-i\left(\gamma^{a}\right)_{\alpha \beta}+\frac{1}{2}\left(\gamma^{e_{1} e_{2}}\right)_{\alpha \beta} X_{e_{1} e_{2} ;}^{a}+\frac{1}{120}\left(\gamma^{e_{1} \ldots e_{5}}\right)_{\alpha \beta} X_{e_{1} \ldots e_{5}}{ }^{a}
$$

and

$$
G_{a b \alpha \beta}=-i\left(\gamma_{a b}\right)_{\alpha \beta}+S_{a b ; e}\left(\gamma^{e}\right)_{\alpha \beta}+\frac{1}{60} S_{e_{1} \ldots e_{5} ;[a}\left(\gamma_{b]}^{e_{1} \ldots e_{5}}\right)_{\alpha \beta}+\ldots
$$

where the ellipses stand for irreps in $G_{2,2}$ other than (11000) and (10002). The BI at dimension 0 reads

$$
\begin{aligned}
0= & \frac{i}{6} \nabla_{a} G_{\alpha_{1} \ldots \alpha_{4}}-\frac{2 i}{3} \nabla_{\left(\alpha_{1} \mid\right.} G_{\left.a \mid \alpha_{2} \alpha_{3} \alpha_{4}\right)}+\frac{2 i}{3} T_{a\left(\alpha_{1} \mid\right.}{ }^{\epsilon} G_{\left.\epsilon \mid \alpha_{2} \alpha_{3} \alpha_{4}\right)} \\
& +\left(\gamma^{f}\right)_{\left(\alpha_{1} \alpha_{2}\right.}\left(\gamma^{e}\right)_{\left.\alpha_{3} \alpha_{4}\right)} S_{f a ; e}+\frac{1}{60}\left(\gamma^{f}\right)_{\left(\alpha_{1} \alpha_{2} \mid\right.}\left(\gamma_{[a \mid} e^{e_{1} \ldots e_{5}}\right)_{\left.\mid \alpha_{3} \alpha_{4}\right)} S_{\left.e_{1} \ldots e_{5} ; \mid f\right]} \\
& +\frac{1}{2}\left(\gamma^{e_{1} e_{2}}\right)_{\left(\alpha_{1} \alpha_{2} \mid\right.}\left(\gamma_{f a}\right)_{\left.\mid \alpha_{3} \alpha_{4}\right)} X_{e_{1} e_{2} ;}^{f}+\frac{1}{120}\left(\gamma^{e_{1} \ldots e_{5}}\right)_{\left(\alpha_{1} \alpha_{2} \mid\right.}\left(\gamma_{f a}\right)_{\left.\mid \alpha_{3} \alpha_{4}\right)} X_{e_{1} \ldots e_{5} ;}{ }^{f}+\ldots
\end{aligned}
$$

Let us denote by $A_{a_{1} a_{2} ; b}^{(1)}, A_{a_{1} a_{2} ; b}^{(2)}$ the irreducible hook-tensor part of the contractions of the rhs of the first line of (86) with

$$
\left(\gamma^{b a}\right)^{\alpha_{1} \alpha_{2}}\left(\gamma^{a_{1} a_{2}}\right)^{\alpha_{3} \alpha_{4}}
$$




$$
\left(\gamma^{b}\right)^{\alpha_{1} \alpha_{2}}\left(\gamma^{\left[a_{1} \mid\right.}\right)^{\alpha_{3} \alpha_{4}} \eta^{\left.\mid a_{2}\right] a}
$$

respectively. We find,

$$
\begin{aligned}
X_{a_{1} a_{2} ; b} & =\frac{-9 A_{a_{1} a_{2} ; b}^{(1)}+2 A_{a_{1} a_{2} ; b}^{(2)}}{2^{12} \times 5} \\
S_{a_{1} a_{2} ; b} & =\frac{3 A_{a_{1} a_{2} ; b}^{(1)}-214 A_{a_{1} a_{2} ; b}^{(2)}}{2^{12} \times 3 \times 5}
\end{aligned}
$$

Similarly, let us denote by $B_{a_{1} \ldots a_{5} ; b}^{(1,2,3)}$, the irreducible hook-tensor part of the contractions of the rhs of the first line of (86) with

$$
\begin{gathered}
\left(\gamma^{b a}\right)^{\alpha_{1} \alpha_{2}}\left(\gamma^{a_{1} \ldots a_{5}}\right)^{\alpha_{3} \alpha_{4}} \\
\left(\gamma^{e}\right)^{\alpha_{1} \alpha_{2}}\left(\gamma_{e}^{a_{1} \ldots a_{5}}\right)^{\alpha_{3} \alpha_{4}} \eta^{a b} \\
\left(\gamma^{b\left[a_{1} \mid\right.}\right)^{\alpha_{1} \alpha_{2}}\left(\gamma^{\left.\mid a_{2} \ldots a_{5}\right] a}\right)^{\alpha_{3} \alpha_{4}}
\end{gathered}
$$

respectively. We find,

$$
\begin{aligned}
X_{a_{1} \ldots a_{5} ; b} & =\frac{4 B_{a_{1} \ldots a_{5} ; b}^{(1)}-3 B_{a_{1} \ldots a_{5} ; b}^{(2)}}{2^{13}} \\
S_{a_{1} \ldots a_{5} ; b} & =\frac{4 B_{a_{1} \ldots a_{5} ; b}^{(1)}-99 B_{a_{1} \ldots a_{5} ; b}^{(2)}}{2^{12} \times 5^{2}} \\
B_{a_{1} \ldots a_{5} ; b}^{(3)} & =\frac{1}{5} B_{a_{1} \ldots a_{5} ; b}^{(1)}-\frac{3}{4} B_{a_{1} \ldots a_{5} ; b}^{(2)}
\end{aligned}
$$

Note that the equation in the last line above must be identically satisfied, if no new constraints are to arise at dimension zero. As a consistency check we have verified that this is indeed the case for the $\nabla_{a} G_{\alpha_{1} \ldots \alpha_{4}}$ terms in (86).

\section{Conclusion}

In this paper we have examined the theory of deformations of $D=11$ supergravity from three different perspectives. We have seen that they can be characterised by elements of certain spinorial cohomology groups with physical coefficients. The zeroth order theory can be presented in terms of $\check{H}_{F}^{1}$ or of $H_{F}^{0,3}$. In the language of [2] these correspond to the groups $\mathcal{H}^{1}, \mathcal{H}^{3}$ of tables 3,4 therein. In the geometrical formulation, the first deformation is given by an element of $\check{H}_{F}^{2}$ (phys), while in the four-form approach it is given as an element of $H_{F}^{0,4}$ (phys). If we include both the four- and seven-forms we have argued that the first deformation, assumed to occur at dimension $\ell^{6}$ and to be at least quartic in the fields, will be uniquely determined by the ChernSimons term needed to cancel the fivebrane anomaly. This will be true if the cohomology groups $H_{\tau}^{p, q}$ all vanish for $p \geq 3$, which we believe to be the case. Given this, all of the components

of the various superspace field strength tensors and the modified action including the $R^{4}$ terms can in principle be constructed systematically.

It should be noted that the uniqueness of the $R^{4}$ invariant in $D=11$ is not purely a result of supersymmetry. We could clearly construct two Chern-Simons terms corresponding to the 
two fourth-order curvature eight-forms which are available and we could expect to be able to supersymmetrise both of them, which would imply that both would appear on the right-hand side of the $G_{7}$ Bianchi identity. However, the fivebrane anomaly will only be cancelled by the correct combination of these two terms and so we must exclude the second superinvariant.

The conclusion that there is just one $R^{4}$ term in M-theory seems to be in line with references $[4,5,7,8]$, but does not agree with [12]. According to [4], in order to obtain the correct anomalycancelling Chern-Simons term, one has to construct a $D=10, N=2$ superinvariant which includes a particular linear combination of the two $D=10, N=1$ superinvariants containing CS terms $[38,39]$, and then lift it to $D=11 .{ }^{4}$ In particular, this implies that the appropriately lifted $t_{8} t_{8} R^{4}$ term should be a partner of the anomaly-cancelling CS term. In reference [12] it is argued that this cannot be the case because CS terms cannot be ultra-violet divergent in quantum supergravity, by a background field method argument, while there is a one-loop divergence in the $t_{8} t_{8} R^{4}$ term $[40,4,5,8]$. However, it is not clear that this argument is correct. ${ }^{5}$ Only the action is required to be gauge-invariant, not the Lagrangian, and this does not seem to rule out divergences of the CS type. The correct CS term does have a finite coefficient in M-theory determined by the anomaly, but it might be the case that this is true even in supergravity. The five-brane occurs as a soliton in the field theory, and it might be the case that quantum supergravity knows about this. If this picture is correct then one could wonder why the one-loop $R^{4}$ term is infinite, but it could be that the calculated divergence is in fact due to curvature terms connected to the "wrong" CS multiplet. On the other hand, it could be the case that all of the coefficients are divergent and that the only thing that perturbative quantum supergravity knows about is the supermultiplet structure. In this case, these coefficients only get fixed to particular finite values (zero for the wrong multiplet) by the anomaly-cancelling mechanism, which could be said to be necessary both in M-theory and in quantum supergravity itself.

The question of whether there can be any non-trivial deformations either with fewer powers of $\ell$ or with fewer than three fields is one that can be tackled and which we hope to address in the near future. In this connection, we note there is another possible Chern-Simons term which could arise at dimension $\ell^{3}$; this would lead to a term of the form $\ell^{3} G_{4} \operatorname{tr} R^{2}$ on the right-handside of the $G_{7}$ Bianchi identity and a Chern-simons term of the form $C_{3} G_{4} \operatorname{tr} R^{2}$. However, such a term is also incompatible with the five-brane anomaly and should therefore be discarded.

We note that, although the formalism allows one to determine the entire theory at order $\beta$ in principle, in practice this would be a very difficult programme to carry through. The lowestdimensional non-vanishing component of the twelve-form $W_{12}$ is $W_{2,10}$ implying that the lowest non-vanishing component of $K_{11}$ is $K_{3,8}$. Proceeding systematically we would therefore need to go through eight Bianchi identity steps to find $K_{11,0}$. If one were to attack the problem this way it would lead to the complete invariant which would include terms of up to eight powers of the gravitino field. Clearly this would be a formidable task. On the other hand, it does not seem

\footnotetext{
${ }^{4}$ The three $N=1, D=10 R^{4}$ invariants discussed in [38, 39] can easily be understood in superspace. There are two involving CS terms with the $B$-field to which can be constructed using the action principle, and the third is a full superspace integral of any function of the dilaton superfield, $\phi$, which is at least quartic in $\phi$.

${ }^{5}$ This is different to the non-renormalisation of the Chern-Simons operator into itself in four-dimensional YangMills theory; in this case the CS operator is a composite operator which appears multiplied by a source in the action.[41]
} 
to be very easy to try to solve the problem starting from the highest dimension and working down. In this case one is nearer to the purely bosonic part of the invariant which is presumably of most interest, but there does not seem to be enough information at first sight to pin it down. This is a problem which needs to be investigated further.

Finally, one can ask whether the formalism could be used, even in principle, to go beyond the first deformation. The term we have found will induce higher-order corrections itself, and these could in principle be determined. A much simpler example of this is given in appendix D in the context of deformations of $D=10$ super Maxwell theory. On the other hand, with more powers of $\ell$ available, one would expect there to be non-vanishing elements in $H_{F}^{0,7}, H_{F}^{1,6}$ and

$H_{F}^{2,5}$ with physical coefficients and the appropriate dimensions. Although one could examine these cohomology groups in principle, in practice it is likely to be prohibitively complicated. One point which might offer some further constraints on the theory is that there is an obstruction to any deformation being integrable to the next order. Again this is briefly discussed for the $D=10$ Maxwell theory in appendix D. In the context of four-form formulation of supergravity the obstruction lies in $H_{F}^{0,5}$ (phys). It might be the case that at least some deformations other than those generated by the fivebrane anomaly are not integrable at higher order, although this would again be difficult to analyse. We note that, at sufficiently high dimension $\left(\partial^{12} R^{4}\right)$ it becomes possible to write down superinvariants as integrals over the whole of $D=11$ superspace. It is not clear that supersymmetry alone places any restrictions on terms of this type.

\section{Acknowledgements}

This work was supported in part by EU contract HPRN-2000-00122 and PPARC grants PPA/G/S/1998/00613 and PPA/G/O/2000/00451.

We would like to thank K. Stelle for informative remarks, particularly concerning Chern-Simons terms. We also thank E. Kiritsis and A. Tseytlin for stimulating discussions.

\section{Appendix A - the $(n, k)$-tensor projection}

Consider the (reducible) $(n, k)$-form, $k \leq n, V_{a_{1} \ldots a_{n} ; b_{1} \ldots b_{k}}$ in $D$ spacetimes dimensions. The projection onto the irreducible part $\hat{\Pi}^{(n, k)} V_{a_{1} \ldots a_{n} ; b_{1} \ldots b_{k}}$ is given by,

$$
\hat{\Pi}^{(n, k)} V_{a_{1} \ldots a_{n} ; b_{1} \ldots b_{k}}=\sum_{l=0}^{k}(-)^{l} \frac{\left(\begin{array}{c}
k \\
l
\end{array}\right)\left(\begin{array}{c}
n+1 \\
l
\end{array}\right)}{\left(\begin{array}{c}
D-n-k+l+1 \\
l
\end{array}\right)} \eta_{a_{1} b_{1}} \ldots \eta_{a_{l} b_{l}} F_{(n, k)}^{l}
$$

where,

$$
F_{(n, k)}^{l}:=\frac{n-k+1}{n+1} \sum_{r=0}^{k-l}\left(\begin{array}{c}
k-l \\
r
\end{array}\right) V^{i_{1} \ldots i_{l}} b_{k-r+1} \ldots b_{k} a_{l+r+1} \ldots a_{n} ; i_{1} \ldots i_{l} b_{l+1} \ldots b_{k-r} a_{l+1} \ldots a_{l+r}
$$

Note that in order to simplify the notation, we have omitted the antisymmetrization brackets. Antisymmetrization is understood in the $\left(a_{1}, \ldots a_{n}\right)$ and $\left(b_{1}, \ldots b_{k}\right)$ indices. The normalization 
is such that $\hat{\Pi}^{2}=1$. The irreducible part $\hat{V}:=\hat{\Pi} V$ constructed above satisfies,

$$
\hat{V}_{\left[a_{1} \ldots a_{n} ; b_{1}\right] b_{2} \ldots b_{k}}=\hat{V}_{a_{2} \ldots a_{n} ; i b_{2} \ldots b_{k}}^{i}=0 \text {. }
$$

In particular we find,

$$
\begin{aligned}
& \hat{V}_{a_{1} a_{2} ; b}=\frac{2}{3}\left(V_{a_{1} a_{2} ; b}+V_{a_{1} b ; a_{2}}\right) \\
& -\frac{1}{5} \eta_{b a_{1}} V_{a_{2} ; i}^{i} \\
& \hat{V}_{a_{1} \ldots a_{5} ; b}=\frac{5}{6}\left(V_{a_{1} \ldots a_{5} ; b}+V_{a_{1} \ldots a_{4} b ; a_{5}}\right) \\
& -\frac{5}{7} \eta_{b a_{1}} V_{a_{2} \ldots a_{5} ; i}^{i} \\
& \hat{V}_{a_{1} a_{2} ; b_{1} b_{2}}=\frac{1}{3}\left(V_{a_{1} a_{2} ; b_{1} b_{2}}+2 V_{a_{1} b_{1} ; a_{2} b_{2}}+V_{b_{1} b_{2} ; a_{1} a_{2}}\right) \\
& -\frac{2}{9} \eta_{b_{1} a_{1}}\left(V_{a_{2} ; i b_{2}}^{i}+V_{b_{2} ; i a_{2}}^{i}\right) \\
& +\frac{1}{45} \eta_{b_{1} a_{1}} \eta_{b_{2} a_{2}} V_{; i j}^{i j} \\
& \hat{V}_{a_{1} \ldots a_{5} ; b_{1} b_{2}}=\frac{2}{3}\left(V_{a_{1} \ldots a_{5} ; b_{1} b_{2}}+2 V_{a_{1} \ldots a_{4} b_{1} ; a_{5} b_{2}}+V_{a_{1} a_{2} a_{3} b_{1} b_{2} ; a_{4} a_{5}}\right)
\end{aligned}
$$

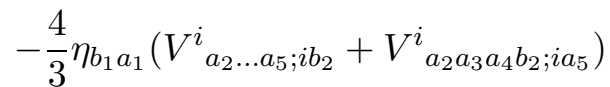

$$
\begin{aligned}
& +\frac{10}{21} \eta_{b_{1} a_{1}} \eta_{b_{2} a_{2}} V^{i j}{ }_{a_{3} a_{4} a_{5} ; i j} \\
& \hat{V}_{a_{1} \ldots a_{5} ; b_{1} \ldots b_{5}}=\frac{1}{6}\left(V_{a_{1} \ldots a_{5} ; b_{1} \ldots b_{5}}+5 V_{a_{1} \ldots a_{4} b_{1} ; a_{5} b_{2} \ldots b_{5}}+10 V_{a_{1} a_{2} a_{3} b_{1} b_{2} ; a_{4} a_{5} b_{3} b_{4} b_{5}}\right. \\
& \left.+10 V_{a_{1} a_{2} b_{1} b_{2} b_{3} ; a_{3} a_{4} a_{5} b_{4} b_{5}}+5 V_{a_{1} b_{1} \ldots b_{4} ; a_{2} \ldots a_{5} b_{5}}+V_{b_{1} \ldots b_{5} ; a_{1} \ldots a_{5}}\right) \\
& -\frac{5}{3} \eta_{a_{1} b_{1}}\left(V_{a_{2} \ldots a_{5} ; i b_{2} \ldots b_{5}}^{i}+4 V_{a_{2} a_{3} a_{4} b_{2} ; i a_{5} b_{3} b_{4} b_{5}}^{i}\right. \\
& \left.+6 V^{i} a_{2} a_{3} b_{2} b_{3} ; i a_{4} a_{5} b_{4} b_{5}+4 V_{a_{2} b_{2} b_{3} b_{4} ; i a_{3} a_{4} a_{5} b_{5}}^{i}+V_{b_{2} \ldots b_{5} ; i a_{2} \ldots a_{5}}^{i}\right) \\
& +\frac{25}{6} \eta_{a_{1} b_{1}} \eta_{a_{2} b_{2}}\left(V^{i j} a_{3} a_{4} a_{5} ; i j b_{3} b_{4} b_{5}\right. \\
& \left.+3 V^{i j}{ }_{a_{3} a_{4} b_{3} ; i j a_{5} b_{4} b_{5}}+3 V^{i j}{ }_{a_{3} b_{3} b_{4} ; i j a_{4} a_{5} b_{5}}+V^{i j}{ }_{b_{3} b_{4} b_{5} ; i j a_{3} a_{4} a_{5}}\right) \\
& -\frac{10}{3} \eta_{a_{1} b_{1}} \eta_{a_{2} b_{2}} \eta_{a_{3} b_{3}}\left(V_{a_{4} a_{5} ; i j k b_{4} b_{5}}^{i j k}\right. \\
& \left.+2 V_{a_{4} b_{4} ; i j k a_{5} b_{5}}^{i j k}+V_{b_{4} b_{5} ; i j k a_{4} a_{5}}^{i j k}\right) \\
& +\frac{5}{6} \eta_{a_{1} b_{1}} \ldots \eta_{a_{4} b_{4}}\left(V_{a_{5} ; i j k l b_{5}}^{i j k l}+V_{b_{5} ; i j k l a_{5}}^{i j k l}\right) \\
& -\frac{1}{21} \eta_{a_{1} b_{1}} \ldots \eta_{a_{5} b_{5}} V_{; i j k l m}^{i j k l m}
\end{aligned}
$$




\section{Appendix B - explicit formulae}

We can use (89) to give the explicit forms of formulae (71-73),

$$
\begin{aligned}
& D A_{a_{1} a_{2} ; b_{1} b_{2}}=A_{a_{1} a_{2} ; b_{1} b_{2}}^{1} \\
& +\frac{1}{2}\left(\gamma_{a_{1}} A_{b_{1} b_{2} ; a_{2}}^{1}+\gamma_{b_{1}} A_{a_{1} a_{2} ; b_{2}}^{1}\right) \\
& +\frac{1}{3}\left(\gamma_{b_{1} b_{2}} A_{a_{1} a_{2}}^{1}+2 \gamma_{b_{1} a_{1}} A_{b_{2} a_{2}}^{1}+\gamma_{a_{1} a_{2}} A_{b_{1} b_{2}}^{1}\right) \\
& D B_{a_{1} \ldots a_{5} ; b_{1} b_{2}}=\frac{2}{3}\left(\gamma_{b_{1} b_{2}} B_{a_{1} \ldots a_{5}}^{1}+2 \gamma_{b_{1} a_{1}} B_{b_{2} a_{2} \ldots a_{5}}^{1}\right. \\
& \left.+\gamma_{a_{1} a_{2}} B_{b_{1} b_{2} a_{3} a_{4} a_{5}}^{1}\right) \\
& +\frac{2}{5}\left(\gamma_{b_{1} b_{2} a_{1}} B_{a_{2} \ldots a_{5}}^{1}-2 \gamma_{b_{1} a_{1} a_{2}} B_{b_{2} a_{3} a_{4} a_{5}}^{1}\right. \\
& +\gamma_{a_{1} a_{2} a_{3}} B_{b_{1} b_{2} a_{4} a_{5}}^{1} \\
& \left.-2 \eta_{a_{1} b_{1}} \gamma_{b_{2}} B_{a_{2} \ldots a_{5}}^{1}-2 \eta_{a_{1} b_{1}} \gamma_{a_{2}} B_{b_{2} a_{3} a_{4} a_{5}}^{1}\right) \\
& +\frac{1}{5}\left(\gamma_{b_{1} b_{2} a_{1} a_{2}} B_{a_{3} a_{4} a_{5}}^{1}+2 \gamma_{b_{1} a_{1} a_{2} a_{3}} B_{b_{2} a_{4} a_{5}}^{1}\right. \\
& +\gamma_{a_{1} a_{2} a_{3} a_{4}} B_{b_{1} b_{2} a_{5}}^{1}-4 \eta_{b_{1} a_{1}} \gamma_{b_{2} a_{2}} B_{a_{3} a_{4} a_{5}}^{1} \\
& \left.+4 \eta_{b_{1} a_{1}} \gamma_{a_{2} a_{3}} B_{b_{2} a_{4} a_{5}}^{1}-\frac{10}{7} \eta_{b_{1} a_{1}} \eta_{b_{2} a_{2}} B_{a_{3} a_{4} a_{5}}^{1}\right) \\
& +\frac{1}{15}\left(\gamma_{b_{1} b_{2} a_{1} a_{2} a_{3}} B_{a_{4} a_{5}}^{1}-2 \gamma_{b_{1} a_{1} \ldots a_{4}} B_{b_{2} a_{5}}^{1}\right. \\
& +\gamma_{a_{1} \ldots a_{5}} B_{b_{1} b_{2}}^{1}-6 \eta_{b_{1} a_{1}} \gamma_{b_{2} a_{2} a_{3}} B_{a_{4} a_{5}}^{1} \\
& \left.-6 \eta_{b_{1} a_{1}} \gamma_{a_{2} a_{3} a_{4}} B_{b_{2} a_{5}}^{1}-\frac{30}{7} \eta_{b_{1} a_{1}} \eta_{b_{2} a_{2}} \gamma_{a_{3}} B_{a_{4} a_{5}}^{1}\right) \\
& +B_{a_{1} \ldots a_{5} ; b_{1} b_{2}}^{1} \\
& +\gamma_{a_{1}} B_{a_{2} \ldots a_{5} b_{1} ; b_{2}}^{1}+\frac{4}{5} \gamma_{b_{1}} B_{a_{1} \ldots a_{5} ; b_{2}}^{1} \\
& +\gamma_{a_{1} a_{2} a_{3}} B_{a_{4} a_{5} ; b_{1} b_{2}}^{1} \\
& -\frac{1}{5}\left(\gamma_{a_{1} \ldots a_{4}} B_{b_{1} b_{2} ; a_{5}}^{1}+\gamma_{a_{1} a_{2} a_{3} b_{1}} B_{a_{4} a_{5} ; b_{2}}^{1}\right. \\
& \left.-\frac{5}{2} \eta_{a_{1} b_{1}} \gamma_{a_{2} a_{3}} B_{a_{4} a_{5} ; b_{2}}^{1}\right) \\
& +\ldots
\end{aligned}
$$




$$
\begin{aligned}
D C_{a_{1} \ldots a_{5} ; b_{1} \ldots b_{5}}= & \frac{1}{6}\left(\gamma_{a_{1} \ldots a_{5}} C_{b_{1} \ldots b_{5}}^{1}+5 \gamma_{a_{1} \ldots a_{4} b_{1}} C_{a_{5} b_{2} \ldots b_{5}}^{1}\right. \\
& +10 \gamma_{a_{1} a_{2} a_{3} b_{1} b_{2}} C_{a_{4} a_{5} b_{3} b_{4} b_{5}}^{1}+10 \gamma_{a_{1} a_{2} b_{1} b_{2} b_{3}} C_{a_{3} a_{4} a_{5} b_{4} b_{5}}^{1} \\
& \left.+5 \gamma_{a_{1} b_{1} \ldots b_{4}} C_{a_{2} \ldots a_{5} b_{5}}^{1}+\gamma_{b_{1} \ldots b_{5}} C_{a_{1} \ldots a_{5}}^{1}\right) \\
+ & C_{a_{1} \ldots a_{5} ; b_{1} \ldots b_{5}}^{1} \\
+ & \frac{1}{4}\left(\gamma_{a_{1} a_{2} a_{3}} C_{b_{1} \ldots b_{5} ; a_{4} a_{5}}^{1}+5 \gamma_{a_{1} a_{2} b_{1}} C_{b_{2} \ldots b_{5} a_{3} ; a_{4} a_{5}}^{1}\right. \\
& \left.+5 \gamma_{a_{1} b_{1} b_{2}} C_{a_{2} \ldots a_{5} b_{3} ; b_{4} b_{5}}^{1}+\gamma_{b_{1} b_{2} b_{3}} C_{a_{1} \ldots a_{5} ; b_{4} b_{5}}^{1}\right) \\
+ & \frac{1}{5}\left(\gamma_{a_{1} \ldots a_{4}} C_{b_{1} \ldots b_{5} ; a_{5}}^{1}+5 \gamma_{a_{1} a_{2} a_{3} b_{1}} C_{b_{2} \ldots b_{5} a_{4} ; a_{5}}^{1}\right. \\
& +10 \gamma_{a_{1} a_{2} b_{1} b_{2}} C_{a_{3} a_{4} a_{5} b_{3} b_{4} ; b_{5}}^{1}+5 \gamma_{b_{1} b_{2} b_{3} a_{1}} C_{a_{2} \ldots a_{5} b_{4} ; b_{5}}^{1} \\
& \left.+\gamma_{b_{1} \ldots b_{4}} C_{a_{1} \ldots a_{5} ; b_{5}}^{1}\right) \\
+ & \ldots
\end{aligned}
$$

\section{Appendix C: Representation-theoretical conventions}

Consider a Lie group $G$ and a $G$-module $V$. The $n$-th tensor product $V^{\otimes n}$ admits a decomposition

$$
\sum_{R} V_{R} \times R
$$

under $G \times S_{n}$, where $R$ runs over all irreducible representations of the symmetric group $S_{n}$ and $V_{R}$ is a $G$-module. As is well known, the irreps of $S_{n}$ can be parametrized by partitions of $n$ or, equivalently, by the associated Young diagrams. If $R$ is associated to the partition $\lambda$ of $n, V_{R}$ is the plethysm of $V$ with respect to $\lambda$.

For the case at hand, the highest weight of the standard module (i.e. of the vector representation) of $\operatorname{Spin}(1,10)$ is given by $(10000)$ on the basis of fundamental weights. A $k$-form is associated to the partition $[1, \ldots, 1]$ of $k$. Its highest weight for $k \leq 4$ is $(0 \ldots 010 \ldots 0)$, with the nonzero entry on the $k$-th position. The five-form is represented by (00002), as the (00001) is the highest weight of the spinor representation. Moreover, the highest weight of the $k$-form-spinor is given by the sum of the highest weight of the $k$-form and the highest weight of the spinor. Recall that by a $k$-form-spinor we refer to the projection onto the irreducible (gamma-traceless) part. For example (10001) is a vector-spinor, (01001) is a two-form-spinor, etc. We can also consider the projection onto the highest-weight representation of the plethysm associated to the partition $[2, \ldots, 2,1, \ldots, 1]$ of $n+k$, with $k$ entries equal to 2 and $n-k$ entries equal to 1. This provides an alternative way to view the $(n, k)$-tensor discussed in Appendix A. Note that $n, k \leq 5$ in the case of $\operatorname{Spin}(1,10)$. The highest weight of an $(n, k)$-tensor with $k, n<5$ is given by $(0 \ldots 010 \ldots 010 \ldots 0)$ where the nonzero entries are on the $k$-th and $n$-th positions. For $k<n=5$ the highest weight is $(0 \ldots 010 \ldots 02)$ with the first nonzero entry on the k-th position. Finally, for $k=n=5$ the highest weight is (00004). In other words, the highest weight of the $(n, k)$-tensor is given by the sum of the highest weight of a $k$-form and the highest weight of an $n$-form. The highest-weights of irreducible (gamma-traceless) $(n, k)$-tensor-spinors are represented in the obvious way. For example (01101) is the highest weight of a $(3,2)$-tensor- 
spinor, (10003) that of a (5,1)-tensor-spinor, etc. This discussion generalizes straightforwardly to representations corresponding to more general partitions.

\section{Appendix D: cohomology}

\section{Pure spinors}

Pure spinors were introduced in $D=10$ super Maxwell theory in an attempt to find an off-shell version [42]. Subsequently it was pointed out that the on-shell constraints of theories in ten and eleven dimensions can be interpreted in terms of pure spinor integrability [43, 19]. A pure spinor in $D=10$ is a complex bosonic chiral spinor $u^{\alpha}$ obeying the single constraint $u^{\alpha}\left(\gamma^{a}\right)_{\alpha \beta} u^{\beta}=0$. Pure spinor integrability implies that $u^{\alpha} u^{\beta} F_{\alpha \beta}=0$ where $F=d A$ is the super Maxwell field strength. In the notation of the current paper this can be written as $\left[F_{0,2}\right]=0$ and this in turn implies that $d_{F}\left[A_{0,1}\right]=0$. Taking gauge transformations this means that the field equations of $D=10$ super Maxwell are encoded in the cohomology group $H_{F}^{0,1}$. Berkovits noticed this in his work and rephrased it slightly differently [44]. He assigns ghost number 1 to the pure spinor $u$ and introduces the BRST operator $Q:=u^{\alpha} D_{\alpha}$ which squares to zero by virtue of the pure spinor constraint. The cohomology group $H_{Q}^{p}$ is defined to be functions $A$ which have ghost number $p$ and which are $Q$-closed modulo $Q$-exact ones. A typical function looks like

$$
A=A_{o}+u^{\alpha} A_{\alpha}+u^{\alpha} u^{\beta} A_{\alpha \beta}+\ldots
$$

where the multispinors in the expansion are all symmetric and gamma-traceless. It is immediately clear that that $H_{Q}^{p}$ is isomorphic to $H_{F}^{0, p}$. In the Maxwell case Berkovits showed that the only non-trivial groups have $p=0,1,2,3$ with $H_{Q}^{0} \sim H_{Q}^{3}$ and $H_{Q}^{1} \sim H_{Q}^{2}$. The elements of $H_{Q}^{1}$ defined the physical fields of the theory as we have seen, and the elements of $H_{Q}^{2}$ can be interpreted as the corresponding anti-fields. We also find that $H_{Q}^{0}=H_{Q}^{3}=\mathbb{R}$ and these two groups are related to the gauge ghost and its anti-field.

\section{Spinorial cohomology}

The notion of spinorial cohomology was introduced independently by the Göteborg group and used in studies of super Yang-Mills and supergravity theories [2, 25, 24]. The concept turns out to be isomorphic to pure spinor cohomology but is presented in a slightly different fashion which is helpful in computing the representations that arise. We illustrate the idea in the $D=10$ Maxwell case. One considers a complex of representations $\left\{r_{k}\right\}$ of the spin group consisting of totally symmetric, gamma-traceless $k$-spinors. (Clearly the same as Berkovits's pure spinor wavefunctions). However, one then tensors each such representation with the $l^{\text {th }}$ antisymmetric power of the fundamental spinor to arrive at an array of representations $\left\{r_{k}^{l}\right\}$. The representations contained in the element $r_{k}^{l}$ are the representations that occur in the $\theta^{l}$ component of a superfield whose leading component is in the representation $r_{k}$. The original set of representations is made into a complex by differentiating a superfield with the spinorial derivative and then projecting onto the gamma-traceless part of the result. Clearly the cohomology obtained this way is isomorphic to pure spinor cohomology if one uses the supercovariant derivative, but if one simply wants to know which representations are present one can use the partial spinorial derivative. It is easy to see that this induces a map $r_{k}^{l} \rightarrow r_{k+1}^{l-1}$, and this fact can be used to compute 
the cohomology using group-theoretic techniques. If we let an element of $r_{k}^{l}$ be denoted by $J_{\alpha_{1} \ldots \alpha_{k}, \beta_{1} \ldots \beta_{l}}$, then the induced operation is

$$
J_{\alpha_{1} \ldots \alpha_{k}, \beta_{1} \ldots \beta_{l}} \mapsto J_{\left\{\alpha_{1} \ldots \alpha_{k}, \beta_{1}\right\} \ldots \beta_{l}} \in r_{n}^{l-1}
$$

where the braces denote gamma-traceless symmetrisation. It is apparent that this defines a linear map $\delta: r_{k}^{l} \rightarrow r_{k+1}^{l-1}$ and that $\delta^{2}=0$. This resembles the Spencer cohomology [45] of a bi-complex $\left\{C_{n, l}\right\}$ of tensors which are symmetric on the first $n$ indices and antisymmetric on the second $l$ with operators $\delta: C_{k, l} \rightarrow C_{k+1, l-1}$ and $\delta^{\prime}: C_{k, l} \rightarrow C_{k-1, l+1}$ defined in the obvious way. In this case since $\delta \delta^{\prime}+\delta^{\prime} \delta=1$ the cohomology is trivial, but in our case this conclusion is avoided by the projection onto the gamma-traceless multi-spinors.

\section{$D=10$ super Maxwell}

To illustrate the meaning of the cohomology groups we again look at $D=10 \mathrm{SYM}$. The two lowest-dimensional components of the Bianchi identity are

$$
\begin{aligned}
& d_{1} F_{0,2}+\tau_{0} F_{1,1}=0 \\
& d_{1} F_{1,1}+\tau_{0} F_{2,0}=0
\end{aligned}
$$

As we have already noted, the on-shell (free) theory is obtained by setting $\left[F_{0,2}\right]=0$ and this is equivalent to specifying an element of $H_{F}^{0,1}$. In this case we note that we can set $F_{0,2}=0$ in which case $F_{1,1}$ determines an element of $H_{F}^{1,1}$. This gives an alternative cohomological way of describing the theory. One can easily see that this cohomology group is a spinor superfield $\lambda^{\alpha}$ satisfying the constraint $D_{\alpha} \lambda^{\beta}=\left(\gamma^{a b}\right)_{\alpha}^{\beta} F_{a b}$, i.e. it is the on-shell field strength supermultiplet. Now consider the group $H_{F}^{0,2}$. We can consider the equation of motion of the theory to be $F_{\{\alpha \beta\}}=0$; if we relax this by introducing a current $J_{\{\alpha \beta\}}$ on the right-hand side then the latter must be spinorially closed by virtue of the Bianchi identity. On the other hand, if it is trivial, the connection can be redefined to regain the original equations of motion. This implies that $H_{F}^{0,2}$ describes the currents of the theory which are in one-to-one correspondence with the antifields. But now suppose that we are interested in looking at deformations of the theory. In this case we need to look at the same cohomology group but the coefficients will be restricted to be tensorial functions of the field strength superfield $\lambda$ and its derivatives. We denote this group by $H_{F}^{0,2}$ (phys). The two groups are quite different; the former is dual to the physical fields while the latter describes a composite multiplet in the theory.

If we deform the theory with respect to a parameter $t$ (in practice $\alpha^{2}$ ) then the above discussion

is relevant to the first deformation. In fact we need only have $d_{F}\left[\stackrel{0}{F}_{0,2}\right]=t\left[\chi_{0,3}\right]+O\left(t^{2}\right)$, where the function $\chi$ is calculated using the first-order corrections to the equations of motion. This term has to be taken into account when we go to second order; it is clearly closed but it also needs to be exact in order for it to be absorbed into the second order deformation. This was observed in [24]. From this discussion it is clear that the possible obstruction to this lies in $H_{F}^{0,3}$ (phys). Provided that the class so defined is trivial, this term, and similar terms at higher order, will give rise to correction terms in the equations of motion, or the component Lagrangian, which are induced by the first deformation. For super Maxwell theory we know that Born-Infeld 
provides a consistent deformation to all orders, so it would be interesting to see how much of the Born-Infeld theory is induced by the first deformation as a result of $N=1$ supersymmetry in ten dimensions. Indeed, it is known that the $F^{6}$ term is induced from the $F^{4}$ term in this way [46].

\section{Spinorial cohomology another way}

Here we give a brief account of spinorial cohomology in superspace in a rather general setting making as few assumptions as possible. Let $M$ denote superspace. We shall assume only that it is equipped with a choice of odd tangent bundle $F$ and that the Frobenius tensor $\varphi$ defined below gives a projection from $\wedge^{2} F$ to the even tangent bundle $B$ which at this stage we can take to be the qotient bundle $T / F$. For any two sections $X, Y \in \Gamma(F)$ the Frobenius tensor is defined by

$$
\varphi(X, Y)=[X, Y] \bmod F
$$

If we introduce a local basis of vector fields $\left\{E_{\alpha}\right\}$ for $F$ and a local basis of one-forms $\left\{E^{a}\right\}$ for the even cotangent space $B^{*}$, the components of $\varphi$ with respect to this basis are essentially the same as those of the dimension-zero component of the torsion tensor.

We let $\Omega^{p}$ denote the space of $p$-forms, and $I$ the ideal of forms generated by $B^{*}$, i.e.the space of all forms which have at least one even index. Set $\Omega_{F}^{p}=\Omega^{p} / I^{p}$ where $I^{p}:=I \cap \Omega^{p}$. This space can be thought of as the space of purely odd (fermionic) $p$-forms. We would like to define an exterior derivative which maps $\Omega_{F}^{p}$ to $\Omega_{F}^{p+1}$ but this is not possible due to the fact that two odd derivatives do not anticommute because of the requirement of supersymmetry. We therefore define the space of reduced odd $p$-forms, $\hat{\Omega}_{F}^{p}$, by

$$
\hat{\Omega}_{F}^{p}:=\Omega^{p} /\left(I^{p} \cup d I^{p-1}\right)
$$

The point of this definition is to get rid of the unwanted terms arising from the anticommutator. It is straightforward to define a derivative $d_{F}$ as follows: let $\left[\omega_{p}\right] \in \hat{\Omega}_{F}^{p}$ where $\omega_{p} \in \Omega^{p}$ is a representative of this equivalence class, then set

$$
d_{F}\left[\omega_{p}\right]:=\left[d \omega_{p}\right]
$$

It is easy to check that this definition is independent of the choice of representative. If $\omega_{p} \mapsto$

$\omega_{p}+\lambda_{p}+d \mu_{p-1}$, with $\lambda_{p} \in I^{p}$ and $\mu_{p-1} \in I^{p-1}$, then $d \omega_{p} \mapsto d \omega_{p}+d \lambda_{p}$. But the second term here is an element of $d I^{p}$ which is factored out in $\hat{\Omega}_{F}^{p+1}$. It is also trivial to see that $d_{F}^{2}=0$.

This construction can be extended to $(p, q)$ forms and to $B$-valued odd forms.

\section{References}

[1] M. J. Duff, "The World In Eleven Dimensions: Supergravity, Supermembranes And MTheory," Bristol, UK: IOP (1999). 
[2] M. Cederwall, B. E. Nilsson and D. Tsimpis, "Spinorial cohomology and maximally supersymmetric theories," JHEP 0202 (2002) 009 [arXiv:hep-th/0110069].

[3] N. Berkovits, "Covariant quantization of the supermembrane," JHEP 0209 (2002) 051 [arXiv:hep-th/0201151].

[4] M. B. Green and P. Vanhove, "D-instantons, strings and M-theory," Phys. Lett. B 408 (1997) 122 [arXiv:hep-th/9704145].

[5] M. B. Green, M. Gutperle and P. Vanhove, "One loop in eleven dimensions," Phys. Lett. B 409 (1997) 177 [arXiv:hep-th/9706175].

[6] M. B. Green, M. Gutperle and H. h. Kwon, "lambda**16 and related terms in M-theory on T**2," Phys. Lett. B 421 (1998) 149 [arXiv:hep-th/9710151].

[7] I. Antoniadis, S. Ferrara, R. Minasian and K. S. Narain, "R**4 couplings in M- and type II theories on Calabi-Yau spaces," Nucl. Phys. B 507 (1997) 571 [arXiv:hep-th/9707013].

[8] J. G. Russo and A. A. Tseytlin, "One-loop four-graviton amplitude in eleven-dimensional supergravity," Nucl. Phys. B 508 (1997) 245 [arXiv:hep-th/9707134].

[9] E. Kiritsis and B. Pioline, "On $\mathrm{R}^{* *} 4$ threshold corrections in type IIB string theory and (p,q) string instantons," Nucl. Phys. B 508 (1997) 509 [arXiv:hep-th/9707018].

[10] N. Berkovits and C. Vafa, "Type IIB R**4 $\mathrm{H}^{* *}(4 \mathrm{~g}-4)$ conjectures," Nucl. Phys. B 533 (1998) 181 [arXiv:hep-th/9803145].

[11] M. B. Green and S. Sethi, "Supersymmetry constraints on type IIB supergravity," Phys. Rev. D 59 (1999) 046006 [arXiv:hep-th/9808061].

[12] A. A. Tseytlin, " $\mathrm{R}^{* *} 4$ terms in 11 dimensions and conformal anomaly of $(2,0)$ theory," Nucl. Phys. B 584 (2000) 233 [arXiv:hep-th/0005072].

[13] K. Peeters, P. Vanhove and A. Westerberg, "Supersymmetric higher-derivative actions in ten and eleven dimensions, the associated superalgebras and their formulation in superspace," Class. Quant. Grav. 18 (2001) 843 [arXiv:hep-th/0010167].

[14] S. de Haro, A. Sinkovics and K. Skenderis, "A supersymmetric completion of the $\mathrm{R}^{* *} 4$ term in IIB supergravity," arXiv:hep-th/0210080.

[15] N. Berkovits and P.S. Howe, unpublished.

[16] E. Cremmer, B. Julia and J. Scherk, "Supergravity Theory In 11 Dimensions," Phys. Lett. B 76 (1978) 409.

[17] E. Cremmer and S. Ferrara, "Formulation Of Eleven-Dimensional Supergravity In Superspace," Phys. Lett. B 91 (1980) 61.

[18] L. Brink and P. S. Howe, "Eleven-Dimensional Supergravity On The Mass - Shell In Superspace," Phys. Lett. B 91 (1980) 384.

[19] P. S. Howe, "Pure spinors, function superspaces and supergravity theories in ten-dimensions and eleven-dimensions," Phys. Lett. B 273 (1991) 90.

[20] P. S. Howe, "Weyl superspace," Phys. Lett. B 415 (1997) 149 [arXiv:hep-th/9707184]. 
[21] M. Cederwall, U. Gran, M. Nielsen and B. E. Nilsson, "Manifestly supersymmetric Mtheory," JHEP 0010 (2000) 041 [arXiv:hep-th/0007035]; "Generalised 11-dimensional supergravity," [arXiv:hep-th/0010042].

[22] M. Cederwall, U. Gran, B. E. Nilsson and D. Tsimpis, in progress.

[23] H. Nishino and S. Rajpoot, "A note on embedding of M-theory corrections into elevendimensional superspace," Phys. Rev. D 64 (2001) 124016 [arXiv:hep-th/0103224].

[24] M. Cederwall, B. E. Nilsson and D. Tsimpis, "Spinorial cohomology of abelian d = 10 super-Yang-Mills at $O\left(\alpha^{\prime 3}\right)$," JHEP 0211 (2002) 023 [arXiv:hep-th/0205165].

[25] M. Cederwall, B. E. Nilsson and D. Tsimpis, "D = 10 super-Yang-Mills at $O\left(\alpha^{\prime 2}\right)$," JHEP 0107 (2001) 042 [arXiv:hep-th/0104236].

[26] M. Cederwall, B. E. Nilsson and D. Tsimpis, "The structure of maximally supersymmetric Yang-Mills theory: Constraining higher-order corrections," JHEP 0106 (2001) 034 [arXiv:hep-th/0102009].

[27] P. S. Howe, E. Sezgin and P. C. West, "Aspects of superembeddings," arXiv:hepth/9705093.

[28] N. Dragon, "Torsion And Curvature In Extended Supergravity," Z. Phys. C 2 (1979) 29.

[29] A. Candiello and K. Lechner, "Duality In Supergravity Theories," Nucl. Phys. B 412 (1994) 479 [arXiv:hep-th/9309143].

[30] L. Bonora, K. Lechner, M. Bregola, P. Pasti and M. Tonin, "A Discussion Of The Constraints In N=1 Sugra-Sym In 10-D," Int. J. Mod. Phys. A 5 (1990) 461.

[31] M. J. Duff, J. T. Liu and R. Minasian, "Eleven-dimensional origin of string / string duality: A one-loop test," Nucl. Phys. B 452 (1995) 261 [arXiv:hep-th/9506126].

[32] R. D'Auria, P. Fre, P. K. Townsend and P. van Nieuwenhuizen, "Invariance Of Actions, Rheonomy And The New Minimal N=1 Supergravity In The Group Manifold Approach," Annals Phys. 155 (1984) 423.

[33] S. J. Gates, M. T. Grisaru, M. E. Knutt-Wehlau and W. Siegel, "Component actions from curved superspace: Normal coordinates and ectoplasm," Phys. Lett. B 421 (1998) 203 [arXiv:hep-th/9711151].

[34] I. A. Bandos, D. P. Sorokin and D. Volkov, "On the generalized action principle for superstrings and supermembranes," Phys. Lett. B 352 (1995) 269 [arXiv:hep-th/9502141].

[35] P. S. Howe, O. Raetzel and E. Sezgin, "On brane actions and superembeddings," JHEP 9808 (1998) 011 [arXiv:hep-th/9804051].

[36] A.M. Cohen, M. van Leeuwen and B. Lisser, LiE v.2.2 (1998), http://wallis.univpoitiers.fr/ maavl/LiE/

[37] U. Gran, "GAMMA: A Mathematica package for performing Gamma-matrix algebra and Fierz transformations in arbitrary dimensions," arXiv:hep-th/0105086.

[38] M. de Roo, H. Suelmann and A. Wiedemann, "Supersymmetric R**4 actions in tendimensions," Phys. Lett. B 280 (1992) 39. 
[39] M. de Roo, H. Suelmann and A. Wiedemann, "The Supersymmetric effective action of the heterotic string in ten-dimensions," Nucl. Phys. B 405 (1993) 326 [arXiv:hep-th/9210099].

[40] E. S. Fradkin and A. A. Tseytlin, "Quantum Properties Of Higher Dimensional And Dimensionally Reduced Supersymmetric Theories," Nucl. Phys. B 227 (1983) 252.

[41] P. Breitenlohner, D. Maison and K. S. Stelle, "Anomalous Dimensions And The AdlerBardeen Theorem In Supersymmetric Yang-Mills Theories," Phys. Lett. B 134 (1984) 63.

[42] B. E. Nilsson, "Pure Spinors As Auxiliary Fields In The Ten-Dimensional Supersymmetric Yang-Mills Theory," Class. Quant. Grav. 3 (1986) L41.

[43] P. S. Howe, "Pure Spinors Lines In Superspace And Ten-Dimensional Supersymmetric Theories," Phys. Lett. B 258 (1991) 141 [Addendum-ibid. B 259 (1991) 511].

[44] N. Berkovits, "Covariant quantization of the superparticle using pure spinors," JHEP 0109 (2001) 016 [arXiv:hep-th/0105050].

[45] D.C. Spencer, "Deformation of structures on manifolds defined by transitive continuous pseudo-groups," Ann. of Math. (2) 76 (1962), 306-445.

[46] A. Collinucci, M. de Roo and M. G. Eenink, "Derivative corrections in 10-dimensional super-Maxwell theory," JHEP 0301 (2003) 039 [arXiv:hep-th/0212012]. 